\title{
Pairwise Trajectory Management (PTM): Concept Overview
}

\author{
Kenneth M. Jones ${ }^{1}$ and Thomas J. Graff ${ }^{2}$ \\ National Institute of Aerospace, Hampton, VA 23666 \\ Ryan C. Chartrand ${ }^{3}$ \\ NASA Langley Research Center, Hampton, VA23681 \\ Dr. Victor Carreño ${ }^{4}$ \\ National Institute of Aerospace, Hampton, VA 23666 \\ and \\ Dr. Jennifer L. Kibler ${ }^{5}$ \\ NASA Langley Research Center, Hampton, VA23681
}

\begin{abstract}
Pairwise Trajectory Management (PTM) is an Interval Management (IM) concept that utilizes airborne and ground-based capabilities to enable the implementation of airborne pairwise spacing capabilities in oceanic regions. The goal of PTM is to use airborne surveillance and tools to manage an "at or greater than" inter-aircraft spacing. Due to the precision of Automatic Dependent Surveillance-Broadcast (ADS-B) information and the use of airborne spacing guidance, the PTM minimum spacing distance will be less than distances a controller can support with current automation systems that support oceanic operations. Ground tools assist the controller in evaluating the traffic picture and determining appropriate PTM clearances to be issued. Avionics systems provide guidance information that allows the flight crew to conform to the PTM clearance issued by the controller. The combination of a reduced minimum distance and airborne spacing management will increase the capacity and efficiency of aircraft operations at a given altitude or volume of airspace. This paper provides an overview of the proposed application, description of a few key scenarios, high level discussion of expected air and ground equipment and procedure changes, overview of a potential flight crew human-machine interface that would support PTM operations and some initial PTM benefits results.
\end{abstract}

\section{Nomenclature}

AC $\quad=$ Aircraft

ADS-B = Automatic Dependent Surveillance-Broadcast

ADS-C = Automatic Dependent Surveillance-Contract

ATC $=$ Air Traffic Control

ATOP $=$ Advanced Technology and Oceanic Procedures

ATS $\quad=$ Air Traffic Services

CDTI $=$ Cockpit Display of Traffic Information

CEP $\quad$ Central East Pacific

CPDLC = Controller-Pilot Data Link Communications

CGD $\quad=$ Configurable Graphics Display

FAA $\quad=$ Federal Aviation Administration

\footnotetext{
${ }^{1}$ Senior Engineer, senior member.

2 Senior Pilot and Controller Subject Matter Expert.

${ }^{3}$ Aerospace Engineer.

${ }^{4}$ Senior Engineer.

${ }^{5}$ Senior Engineer.
}

American Institute of Aeronautics and Astronautics 


$\begin{array}{ll}\text { FIM } & =\text { Flight Deck Interval Management } \\ \text { FL } & =\text { Flight Level } \\ \text { FMC } & =\text { Flight Management Computer } \\ \text { FOV } & =\text { Field-of-View } \\ \text { HF } & =\text { High Frequency radio } \\ \text { HMI } & =\text { Human-Machine Interface } \\ \text { IM } & =\text { Interval Management } \\ \text { MCP } & =\text { Mode Control Panel } \\ \text { NASA } & =\text { National Aeronautics and Space Administration } \\ \text { NM } & =\text { Nautical Miles } \\ \text { PCD } & =\text { Projected Closest Distance } \\ \text { PTM } & =\text { Pairwise Trajectory Management } \\ \text { SLOP } & =\text { Strategic Lateral Offset Procedure }\end{array}$

\section{Introduction}

Interval Management (IM) is a set of capabilities designed to support a range of IM operations and concepts whose goal is precise inter-aircraft spacing. IM is defined as the overall system, including both ground and airborne domains, that enables improved management of traffic flows, aircraft spacing and separation assurance. This includes both the use of ground and airborne tools, where ground tools assist the controller in evaluating the traffic picture and determining appropriate clearances to space aircraft efficiently and safely, and airborne tools that allow the flight crew to conform to the clearance.

Pairwise Trajectory Management (PTM) is an IM concept that utilizes airborne and ground-based tools to enable airborne inter-aircraft spacing in oceanic regions. The goal of PTM is to use airborne surveillance and tools to manage "at or greater than" inter-aircraft spacing. Due to the Automatic Dependent Surveillance-Broadcast (ADS-B) information and the use of airborne spacing guidance, the PTM minimum spacing distance will be less than distances a controller can support with current automation systems that support oceanic operations. Ground tools assist the controller in evaluating the traffic picture and determining appropriate PTM clearances to be issued. Avionics systems provide guidance information that allows the flight crew to conform to the PTM clearance issued by the controller. The combination of a reduced minimum distance and airborne spacing management will increase the capacity and efficiency of aircraft operations at a given altitude or volume of airspace.

PTM was designed to improve operations that can occur in a variety of oceanic environments and situations. These oceanic regions are characterized by reduced surveillance and limited communication capabilities. In such airspace, air traffic is managed procedurally with reliance on specified navigation performance, periodic position reports to the ground, and separation standards that are larger than commonly applied in domestic airspace. These airspace regions also involve a variety of route structures including fixed routes, organized track system routes, and user preferred routes. This document describes a number of generic PTM operations and scenarios. These scenarios were used to explore the operational requirements needed to support PTM operations.

NASA has been developing the PTM concept for a number of years ${ }^{1}$. This paper provides an updated overview of the proposed application, description of key scenarios, a high level discussion of expected air and ground equipment and procedure changes, some initial benefits analyses and the description of a NASA human-machine interface (HMI) prototype for the flight crew that would support PTM operations.

\section{Application Description}

Studies indicate there will be continued growth in oceanic air traffic. Even with recent improvements to oceanic surveillance and communications systems, required separation standards in oceanic airspace are still large enough to cause aircraft operational inefficiencies. These inefficiencies include operating at other than their desired altitude, routes, and speeds for extended periods of time, flights being unable to operate on their desired routes, and flights required to change altitudes for crossing traffic. These system inefficiencies increase flight time and fuel burn.

PTM is designed to enable operations that are not possible with current non-PTM separation standards. These operations will be enabled by more efficient spacing assignments issued by controllers and managed by aircraft. The controller, using ground-based decision-support automation, issues a pair-specific PTM clearance to a PTM-equipped aircraft. When accepted, this clearance will allow the PTM aircraft to operate on the same flight level and track of the designated aircraft (the paired aircraft), or to cross the track of the designated aircraft with reduced spacing as compared to current non-PTM separation standard minimums. The PTM clearance requires the flight crew of the PTM

American Institute of Aeronautics and Astronautics 
aircraft to use their ADS-B-enabled on-board Flight Deck Interval Management (FIM) equipment to manage their spacing relative to the designated aircraft (the term "FIM Equipment" in this document is used to refer to any avionics on board the PTM aircraft that supports the PTM application). The FIM equipment will provide speed guidance to ensure that the inter-aircraft spacing is no closer than the PTM minimum distance during the PTM operation. When the controller assesses (with ground automation system support) that a non-PTM separation standard has been achieved and the PTM operation is no longer required, the controller may terminate the PTM operation. The controller is not required to terminate the PTM operation as soon as non-PTM separation standards are available and may allow the PTM operation to continue for operational reasons.

PTM will support same track operations (typically found in oceanic organized track systems) and crossing track operations (typically found with oceanic user preferred routes). Due to the reduced PTM minimum spacing distance, the PTM aircraft may not be required to modify its current speed in many traffic crossing situations. PTM should save fuel and reduce delays by improving operations that increase time on an aircraft's optimal trajectory (track, speed and altitude). The airborne managed distance can allow for higher throughput and generally more efficient aircraft operations.

PTM may be employed, depending on local constraints and traffic characteristics, to support several different types of operations. For example, a PTM clearance could be used to resolve a short-term conflict (e.g., one aircraft climbing through the altitude of another) or could be used for an extended period of time (same track, same altitude).

The availability of a PTM resolution to a conflict does not necessarily make it the preferred option for the controller. PTM is not intended to take away the controller's discretion in managing the traffic in their sector; PTM is simply an additional tool for the controller to use as needed or desired.

There are also times when PTM is not an available option for the controller. One example of this is when the two aircraft are out of ADS-B range from each other (since the PTM aircraft must be receiving the ADS-B signal of the designated aircraft to accept the clearance and manage spacing). Additionally, other operational conditions may cause the flight crew to refuse the PTM clearance. This could include situations when PTM operations would require the flight crew to fly a speed that is faster or slower than the aircraft can maintain. In addition, certain traffic encounter geometries may prevent the availability of PTM to be used by either the controller or the flight crew (e.g., head on traffic encounter geometries).

The following sections include descriptions of two typical PTM scenarios that illustrate details of the PTM concept. For all graphic depictions in this document, the following applies for planform and profile views of aircraft:

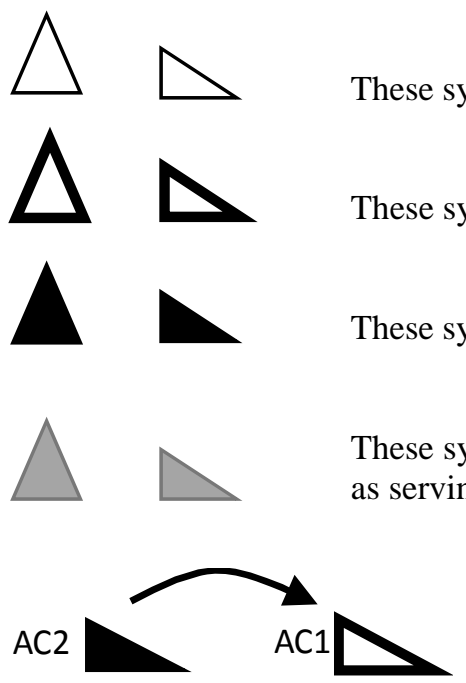

These symbols are directional, and the color indicates background aircraft

These symbols are directional, and the bold border indicates designated aircraft

These symbols are directional, and the color indicates PTM aircraft.

These symbols are directional and the color indicates the aircraft are PTM aircraft as well as serving as a designated aircraft for another PTM aircraft.

These symbols indicate aircraft 2 (AC2) is a PTM aircraft and aircraft 1 (AC1) is the designated aircraft for the PTM operation that AC2 is conducting.

\section{A. Same-Track, Altitude Change Scenario}

Figure 1 is a profile view representation of two aircraft (AC1 and AC2) operating at different altitudes on an identical track with a distance between them of 9 nautical miles (NM). In this scenario, the crew of AC2 has requested, for operational reasons, an altitude change from Flight Level 330 (FL330) to FL340. Using non-PTM separation standard distances, the requested climb would be denied due to traffic (AC1) at FL340. However, because AC2 has indicated in its flight plan that it is PTM equipped, the ground automation system would indicate the possibility of issuing a PTM clearance to AC2, which if accepted, would allow the controller to subsequently issue a climb clearance.

American Institute of Aeronautics and Astronautics 


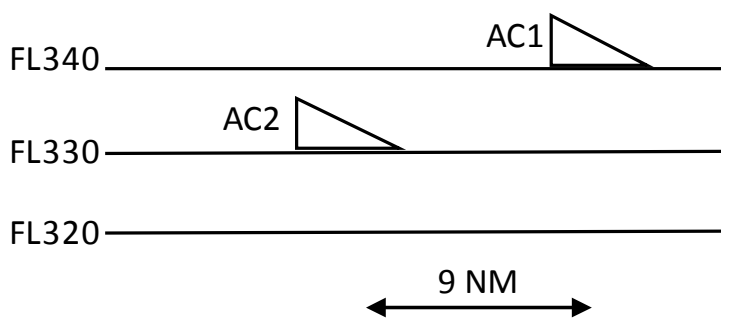

Figure 1. Same-Track, Altitude Change Scenario

If PTM is acceptable from a controller's perspective, the controller would issue a PTM clearance to AC2 instructing it to conduct PTM on AC1. Once the clearance is received by AC2 (the PTM aircraft), the PTM clearance information would be loaded into the FIM equipment. The FIM equipment would check the acceptability of the PTM clearance and display PTM speed guidance the flight crew would be expected to follow should they accept the clearance. The flight crew would review the proposed PTM speed guidance (fixed Mach speed or Mach speed range) to determine if the proposed guidance would be operationally acceptable. The flight crew would also review other information that could influence their decision to accept or reject the PTM clearance. This would include assessing potential future speed restrictions based on the current speeds of the designated aircraft (e.g., the eventual speed the PTM aircraft may be required to fly due to the speed of the designated aircraft). Other information that could influence the flight crew's decision would include aircraft performance, weather conditions, available ride information and general traffic situation awareness.

If the proposed guidance and clearance are acceptable, the flight crew would engage the FIM equipment and accept the clearance. With the PTM operation engaged, a single speed or a speed range is continuously displayed by the FIM equipment to the flight crew. If, instead, the proposed guidance or situation is unacceptable, the flight crew clears the proposed PTM guidance in the FIM equipment, rejects the clearance with ATC and continues normal operations.

Once the controller has received confirmation that the PTM clearance has been accepted, the controller would then clear AC2 to climb to and maintain FL340. The PTM aircraft would then start a normal climb to FL340. The flight crew follows the speed guidance provided by the FIM equipment until the PTM operation is terminated by the controller. Figure 2 below is an illustration of the situation once the climb to FL340 has been completed.

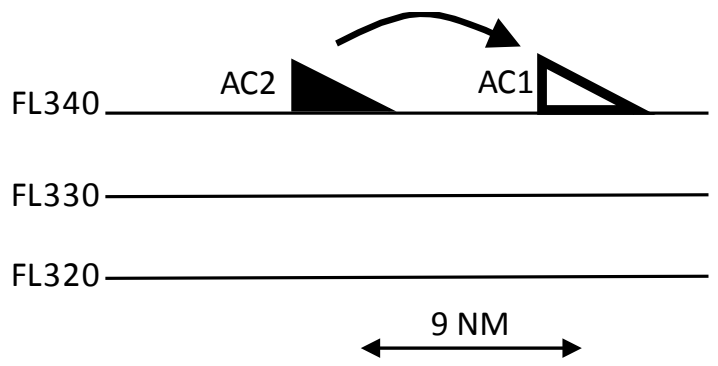

Figure 2. Same-Track, Altitude Change Scenario

Messages have been developed for PTM and are included in recently published versions of RTCA documents DO-350, DO-351 and DO-352. These documents contain the standards that have been adopted for Baseline 2 ATS Data Communications.

The combination of messages used to support PTM operations is called a message queue. The message queue consists of a list of Controller Pilot Data Link Communications (CPDLC) messages showing the destination, the sender, and the message. The CPDLC application allows addressed communications (only the intended aircraft receives the message) between the ground controller and the flight crew, to provide air traffic services.

Due to the complexity and importance of the structure of these messages, it is anticipated that the logic for generating these message queues would be built into ground automation systems used to support PTM operations.

A sample of communications for this scenario is listed below. The format of these messages includes the recipient of the message (the destination) and who is sending the message (sender).

American Institute of Aeronautics and Astronautics 
Message Queue [Destination, Sender]

[ATC, AC2] REQUEST CLIMB TO FL340

[AC1, ATC] MAINTAIN MACH 0.83

[ATC, AC1] WILCO

[AC2, ATC] CLEARED PTM MACH BEHIND AC1, LIMIT MACH FOR PTM OPERATION 0.83

[ATC, AC2] WILCO

[AC2, ATC] CLIMB TO AND MAINTAIN FL340, REPORT REACHING FL340

[ATC, AC2] WILCO

[ATC, AC2] LEVEL FL340

\section{B. Intersecting Track Scenario}

There will be cases where PTM will be used to support intersecting track situations. For the intersecting track situation shown in figure 3 below, AC2 and AC1 are moving towards a loss of separation situation in an oceanic environment with a $30 \mathrm{NM}$ minimum separation distance. The aircraft are both at FL310 and Mach 0.80. Without PTM, the controller would need to instruct one of the two aircraft to change its altitude or speed. However, because AC2 has indicated that it is PTM equipped in its flight plan, the ground automation system would indicate the possibility of issuing a PTM clearance for the controller. The controller can use a PTM clearance to resolve the conflict. If PTM is acceptable from the controller's perspective, the controller would issue a clearance to AC2 instructing it to conduct PTM on AC1.
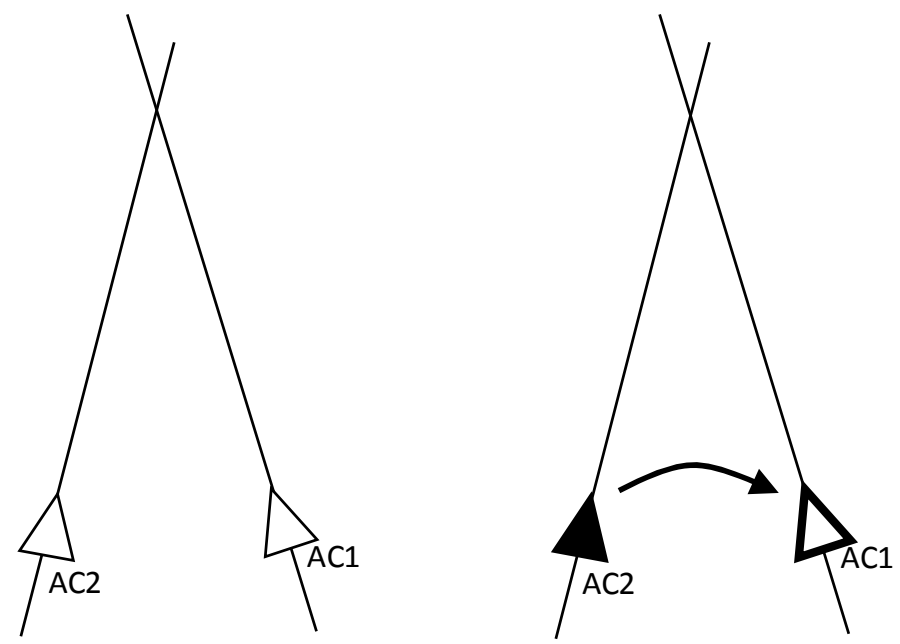

Figure 3. Intersecting Tracks Scenario prior to (on the left) and after (on the right) PTM is engaged

When the PTM clearance is received by AC2, the PTM clearance information would be loaded into the FIM equipment. The acceptability of the PTM clearance would be checked against associated criteria required for PTM operations. The FIM equipment uses data received from AC1 and the ground automation system to predict the time, location, and distance at the closest point of approach. The system displays Mach guidance to the flight crew that would ensure the required spacing between the PTM aircraft and the designated aircraft is satisfied during the PTM operation.

As before, the flight crew would review the proposed PTM speed guidance (fixed Mach speed or Mach speed range) to determine if the proposed guidance would be operationally acceptable. The flight crew would also review other information that could influence their decision to accept or reject the PTM clearance. This would include assessing potential future speed restrictions (e.g., the eventual speed the PTM aircraft may be required to fly due to the speed of the other aircraft) based on the current speed of the designated aircraft. Other information that could influence the flight crew's decision would include aircraft performance, weather conditions, available ride information and general traffic situation awareness.

If the proposed guidance was acceptable, the flight crew would engage the FIM equipment and accept the clearance. It is likely that, while the aircraft will pass too close for a non-PTM separation standard, the aircraft will be significantly farther apart than required for the PTM separation standard. The flight crew follows the speed guidance provided by the FIM equipment throughout PTM operations. Once the aircraft have passed the closest point of

American Institute of Aeronautics and Astronautics 
approach and the controller determines that a non-PTM separation standard has been achieved (e.g., $30 \mathrm{NM}$ ), the controller may terminate the PTM procedure.

A sample of the messages used for this scenario are listed below:

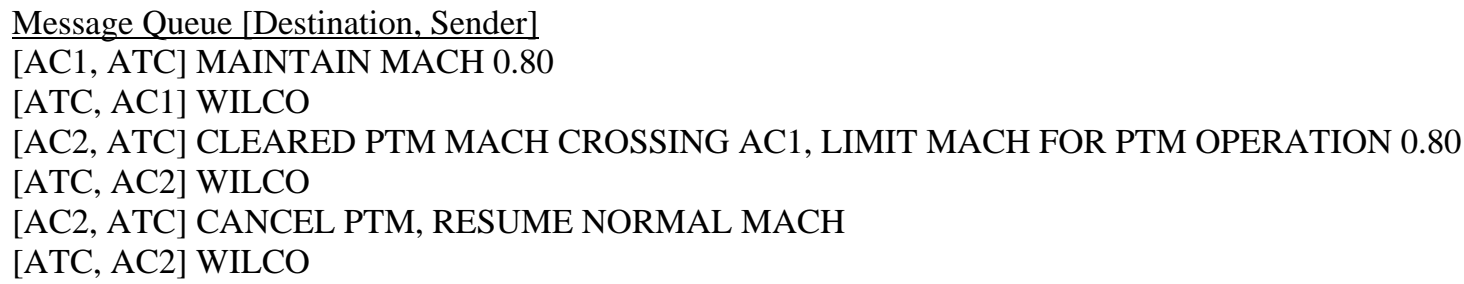

\section{Assumptions and Constraints}

There are several key assumptions and constraints associated with PTM operations. These are outlined below.

\section{A. PTM Separation Standard and PTM Spacing Distance}

A key component of PTM is a reduced minimum distance, referred to as the PTM separation standard distance, enabled by active ADS-B In monitoring and real-time, closed-loop interval management control. The PTM separation standard distance is a horizontal distance that the PTM aircraft must not get closer than during PTM operations. PTM aircraft can be at distances that are greater than this minimum distance, but the horizontal distance between a PTM aircraft and all designated aircraft must never be less than the PTM separation standard distance while a PTM operation is in effect. The PTM separation standard distance is a horizontal distance and is applicable in along-track as well as intersecting track operations. This PTM separation standard distance will be determined by safety and performance analyses. Initial analyses indicate this distance could be set at $5 \mathrm{NM}$.

The PTM spacing distance is the PTM separation standard distance plus a buffer. The size of the buffer is operation specific. The proposed buffer is $1.5 \mathrm{NM}$ for along track operations and 3.5 NM for intersecting track operations. This results in a total PTM spacing distance of 6.5 or $8.5 \mathrm{NM}$. The intent of this buffer is not to imply precise management of the PTM spacing distance or to drive the PTM aircraft to a specific spacing objective, but to ensure that the PTM separation standard distance is not violated for wide-ranging operational behaviors and situations. As with the PTM separation standard distance, the final magnitude of these values will be determined by safety and performance analyses.

\section{B. Along-Track PTM Spacing Distance}

When aircraft are on the same identical track, a spacing buffer must be applied to ensure that aircraft do not get closer than the PTM separation standard distance during normal operations. One typical scenario that demonstrates the importance of this buffer is when two aircraft are on the same route and the route includes a turn at a waypoint. In situations such as these, the along-track distance will remain the same but the absolute range will be reduced below the along-track range.

This is shown in figure 4 below where the PTM separation standard distance is assumed to be 5 NM and the PTM spacing distance is $6.5 \mathrm{NM}$ (i.e., a $1.5 \mathrm{NM}$ buffer). Prior to the turn at the waypoint, both aircraft have an absolute range and along track range of $6.5 \mathrm{NM}$. Once the aircraft are on opposite sides of the turn, the absolute range will be less than 6.5 $\mathrm{NM}$ but the along track range will remain at 6.5 NM.

The extent of this buffer affects the acceptable geometries for feasible PTM operations, generally limiting turn angles to no greater than 45 degrees.

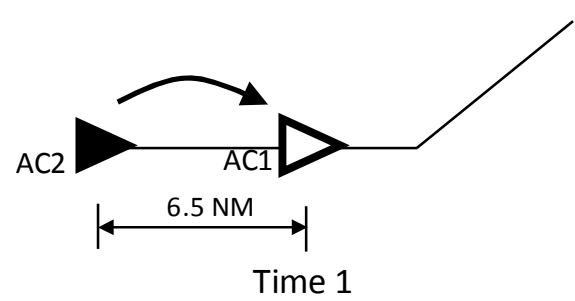

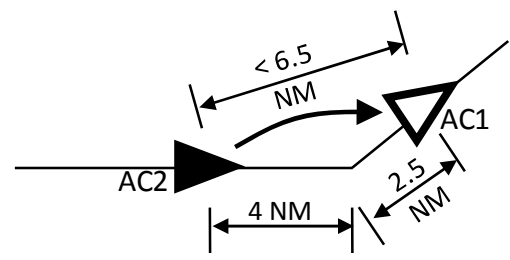

Time 2

Figure 4. Along Track PTM Spacing Distance

American Institute of Aeronautics and Astronautics 


\section{Intersecting Track PTM Spacing Distance}

Similar to the along-track scenario, the specific geometry of the operation must be considered when determining the PTM spacing needed to ensure the 5 NM PTM separation standard distance is not violated. The same nominal buffer of $1.5 \mathrm{NM}$ would apply to these geometries as well. However, the crossing case requires an additional buffer to account for when both aircraft are on a Strategic Lateral Offset Procedure (SLOP). The possible SLOP combinations at SLOP operations of up to $2 \mathrm{NM}$ route offset to the right could lead to an error in the position of the assumed crossing point as depicted in figure 5 below.

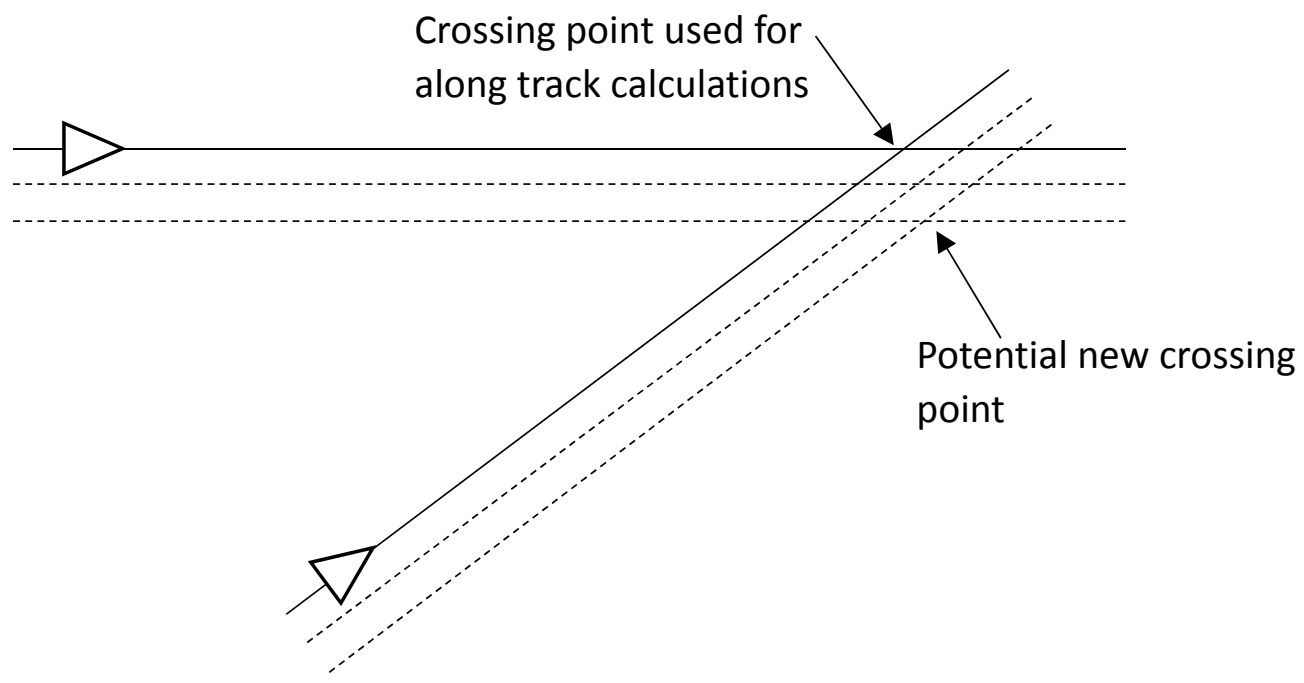

Figure 5. Intersecting track PTM spacing distance considerations

This error is accounted for by an additional 2 NM buffer bringing the total buffer to 3.5 NM and the total PTM spacing to $8.5 \mathrm{NM}$. It should be noted that the effect of SLOP in same-track operations is accounted for in the original 1.5 NM buffer, but the potential difference in path length from SLOP combinations is much smaller. Similar to the along-track scenario, the acceptability of this 8.5 NM PTM spacing is predicated on assumed geometries, such as intersection angles of no greater than 45 degrees.

Importantly, since ensuring this PTM separation is the operational intent, the PTM spacing objective is assessed as a horizontal range between the aircraft at an estimated point of closest approach.

\section{PTM Operations during Altitude Change Maneuvers}

There are scenarios (as demonstrated in section II.A above) where PTM is used to enable altitude change maneuvers. These include situations where an aircraft desires a change to the same altitude of another aircraft or where an aircraft is flying "through" the altitude of another aircraft.

The first step in this procedure is to establish PTM operations with the designated aircraft. This is to ensure that during altitude change maneuvers, there is valid longitudinal and lateral separation between the PTM aircraft and the designated aircraft before vertical separation is lost and throughout the ensuing PTM operation. Once PTM is established the altitude change clearance can be issued.

It is important to point out that with PTM, FIM equipment will provide PTM Mach guidance to manage spacing with designated aircraft, even if those aircraft are at different altitudes. This is because the PTM separation standard is a longitudinal and lateral separation standard and takes no "credit" for vertical separation.

In the illustration below (figure 6), for an aircraft climbing, PTM is first established between AC2 and AC1. Once engaged, the FIM equipment will provide PTM Mach guidance that will enable AC2 to manage spacing with AC1 even while AC2 remains at the original altitude. Once the controller has received a reply from the flight crew that PTM has been established between AC2 and AC1, AC2 is cleared to climb. AC2 then climbs to the desired altitude and continues to follow the PTM Mach guidance.

American Institute of Aeronautics and Astronautics 

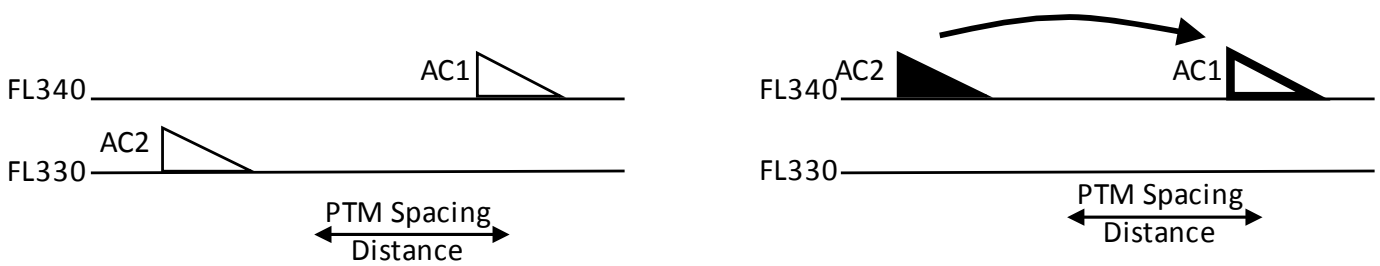

Figure 6. PTM operations during altitude change maneuvers

\section{E. Multiple PTM Operations within a non-PTM Separation Distance}

When there are multiple aircraft performing PTM in a sequence within a non-PTM separation standard distance, some special rules apply. In this case, PTM must be conducted on all aircraft that are within the non-PTM separation standard distance. This ensures there is an appropriate separation standard being used between all pairs of aircraft.

In the illustration in figure 7 below, PTM aircraft AC2 is conducting PTM on designated aircraft AC1. PTM aircraft AC3 is conducting PTM operations on AC2 (in the direction of the designated aircraft AC1). However, because AC3 and AC1 are within the minimum non-PTM separation standard distance, AC3 must also conduct PTM on the designated aircraft AC1. Without this addition, there is no applicable separation between AC3 and AC1.

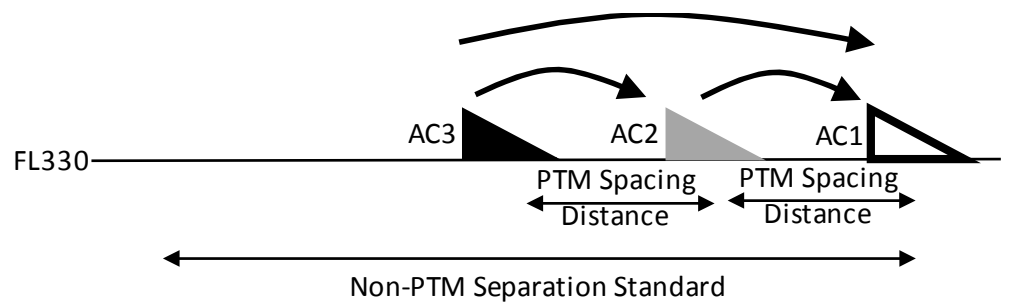

Figure 7. Multiple PTM operations within a non-PTM Separation Distance

\section{F. “Separation by Order"}

It has been recommended that the concept for the PTM separation standard be revised to include the concept of “separation by order”. Currently, the proposed PTM separation standard does not account for the fact that aircraft AC2 is between AC3 and AC1. Rather than require AC3 to conduct PTM on AC1, the revised PTM separation standard would only require AC3 to conduct PTM on AC2. AC3 would be separated from AC1 by virtue of the fact that AC2 is between the two aircraft and that AC3 would get too close to AC2 before it would get too close to AC1; essentially taking advantage of the order the aircraft.

If this revision to the proposed PTM separation standard was adopted, PTM aircraft would only need to conduct PTM operations on aircraft that are closest to them (as depicted in figure 8). This would simplify PTM operations, the flight crew's HMI and the PTM message queues.

Prior to adopting this simplification, a thorough, updated safety analysis must be performed. One advantage of the current approach is that if AC2 has a problem and must suddenly exit out of the string (e.g., if AC2 has an engine failure), there is already a PTM operation in place between AC3 and AC1. With the revised concept, if AC2 leaves a string, a PTM operation would have to be initiated between AC3 and AC1. Due to the relatively small speed differences (low closure rates) between aircraft operating in oceanic airspace regions, there should be sufficient time to apply PTM to these aircraft before they get closer to each other than the minimum PTM spacing distance.

"Separation by Order" is not assumed for the purposes of this paper.

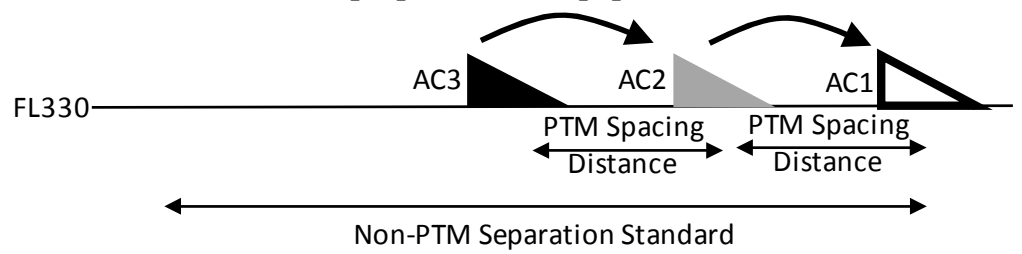

Figure 8. PTM operations including the separation by order revision to the PTM Separation Standard

American Institute of Aeronautics and Astronautics 


\section{G. PTM Algorithm}

A PTM algorithm has been developed to provide part of the airborne functionality needed to perform PTM operations. There is a top-level module named the executive module. The executive module receives PTM clearances from ATC and using the PTM operations criteria and other information, advises the crew whether the PTM clearance should be accepted or rejected. If the flight crew accepts the PTM clearance, the executive module is invoked continuously to provide speed guidance to the flight crew to ensure appropriate spacing from the designated aircraft. The executive module also receives clearances to add designated aircraft to an existing PTM operation and to terminate PTM operations with existing designated aircraft.

Other modules and functions that comprise the airborne PTM algorithm include the Along Track module, the Intent Speed Resolution module, and the Alert function. The Along Track Module calculates the speed guidance using a state projection of the ownship and designated aircraft. The Intent Speed Resolution module calculates a speed guidance using the current location, the next waypoints, the next-plus-one waypoints, and the estimated time of arrivals to the waypoints of the ownship and designated aircraft. The alert function produces predicted, imminent, and actual loss of spacing alerts. The executive module selects between the Along Track and Intent Speed Resolution modules to produce the speed guidance. Selection is determined by the type of clearance, geometry, waypoint locations, and other criteria.

\section{Calculation of along track PTM Mach guidance}

The along track algorithm generates Mach guidance that, if followed, will ensure that the inter-aircraft spacing is no closer than the PTM minimum distance during the PTM operation. The objective of the algorithm is for the PTM aircraft to manage spacing from the designated aircraft in a safe and efficient manner.

The spacing is the sum of a separation standard minimum distance and buffer distance. The algorithm provides speed guidance to achieve the spacing. If the along track distance is greater than the spacing, the algorithm could give speed guidance such that the along track distance can be reduced. When the along track distance is at or near the spacing, the algorithm will give speed guidance to maintain the spacing.

An example of how this works can be seen in the following example. In figure 9 below, AC1 is a designated aircraft for PTM aircraft AC2. AC1 is flying at a Mach speed of $\mathrm{M}=0.80$. AC2 is flying at $\mathrm{M}=0.75$ and for operational reasons, AC2 can fly no slower than M=0.75. AC2 is currently $25 \mathrm{NM}$ away from AC1. Since AC2 is behind AC1, the along track algorithm will calculate an upper speed guidance that AC2 can fly.

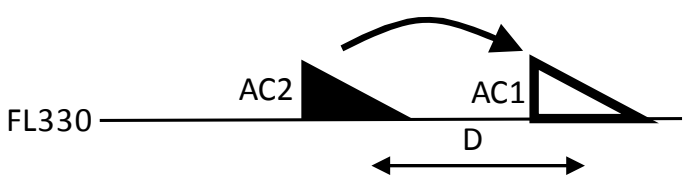

Figure 9. Closing PTM aircraft behind a designated aircraft

For this situation, the along track algorithm will provide an upper guidance of $\mathrm{M}=0.86$. At this distance, the flight crew would be provided a PTM Mach guidance range of $M=0.75$ (the lower bound) to $M=0.86$ (the current algorithm calculated upper bound). As a result, if the flight crew chose to fly at the upper bound of $M=0.86$, AC2 would close to a distance of $6.5 \mathrm{NM}$ with AC1 in 30 minutes. However, as AC2 gets closer to AC1, the Mach guidance will change and the upper speed guidance will be less than $\mathrm{M}=0.86$.

The Mach guidance will be updated as the operation continues. This is done in increments of $M=0.01$. At each update, the upper Mach guidance will ensure that it would take at least 30 minutes to close the distance to $6.5 \mathrm{NM}$. However, once the PTM Mach guidance is within $M=0.01$ of the designated aircraft's Mach (in this case $M=0.80$ ), the upper Mach guidance would stay $\mathrm{M}=0.01$ above the designated aircraft Mach until the two aircraft are within 6.5 NM (with hysteresis effects built into the logic to minimize short-term fluctuations in the Mach guidance). Table 1 contains sample Mach guidance that could be provided to the flight crew for various distances between the two aircraft.

\begin{tabular}{|l|l|l|l|}
\hline D & $\mathbf{2 5} \mathbf{~ N M}$ & $\mathbf{7 ~ N M}$ & $\mathbf{6 . 5} \mathbf{~ N M}$ \\
\hline $\begin{array}{l}\text { Upper bound Mach } \\
\text { guidance }\end{array}$ & $\mathrm{M}=0.86$ & $\mathrm{M}=0.81$ & $\mathrm{M}=0.80$ \\
\hline $\begin{array}{l}\text { Lower bound Mach } \\
\text { guidance }\end{array}$ & $\mathrm{M}=0.75$ & $\mathrm{M}=0.75$ & $\mathrm{M}=0.75$ \\
\hline
\end{tabular}

Table 1. Changes to Mach guidance based on distance to designated aircraft

American Institute of Aeronautics and Astronautics 


\section{Flight crew input of operating Mach number range}

The default maximum and minimum possible values of Mach numbers provided to flight crews during operations would be set based on the maximum and minimum possible values the aircraft is capable of flying. During initial studies, flight crews have expressed a desire to have a method to input the operating Mach range they are able and/or willing to fly at a given time. The algorithm is designed in such a way that it would permit the flight crew to enter minimum and maximum possible values at any time during the flight. If the flight crew is currently conducting PTM operations, the current PTM Mach guidance must be contained within the minimum and maximum bounds set by the flight crew.

\section{H. ADS-B Signal Range Impact on PTM Operations}

For oceanic operations, there are widely varying separation standards applicable for the controller to use with a specific aircraft pair. These typically vary from as small as $30 \mathrm{NM}$ to as large as approximately $120 \mathrm{NM}$ (based on a 15 minute separation standard) between the aircraft. It should be noted that there is other work being done to further reduce these standards and these should be considered as they are approved. During normal operations, most ground automation systems report projected losses of separation (traffic conflicts) and often display which separation standards may be available to the controller to solve the traffic conflict. If the ground automation system reports that the conflict is with a PTM aircraft and a suitable designated aircraft, PTM would be one of the potential options listed to the controller.

It is possible that at the time the conflict is first reported by the ground system, the onboard FIM equipment may not be receiving a signal from the designated aircraft identified due to ADS-B signal range limitations. Typically, ADS-B signals are received up to an average of $180 \mathrm{NM}$ away. If the two aircraft are at a distance that is greater than a nominal ADS-B signal range at the time the PTM clearance was issued, it is likely that the flight crew would not be able to identify the designated aircraft and it would be necessary for the flight crew to refuse the clearance.

To maximize acceptance of PTM clearances, the controller should wait until the ground automation system computes that the two aircraft are close enough to each other (within an expected ADS-B range) so that there is a high probability that the FIM equipment will be able to identify the designated aircraft and enable acceptance of the PTM clearance. This expected ADS-B range could be a facility configurable parameter that could be adjusted as experience with ADS-B signal range is obtained.

Another aspect of operations that would go into this decision-making process is the time or distance prior to a predicted loss of separation when the controller needs to issue a clearance. This is often determined either by local policy and/or controller comfort levels. For example, in most US managed oceanic airspace, controllers are notified by ground automation of a conflict two hours prior to the loss of separation and should be in the process of resolving the conflict at least 30 minutes prior to the predicted loss of separation.

As a result, the nominal ADS-B range (approximately $180 \mathrm{NM}$ ) and time prior to loss of separation that traffic conflicts are resolved impacts the use of PTM operations. This is particularly true for intersecting track geometries.

If a controller has a conflict with two aircraft with a $30 \mathrm{NM}$ separation standard, and the controller wants to issue a clearance 40 minutes prior to the predicted loss of separation in an environment with a $180 \mathrm{NM}$ expected ADS-B range, the crossing angle for PTM would be limited to approximately 30 degrees. This can be illustrated in the following example.

In figure 10 below the two aircraft are about 40 minutes away from a loss of separation (both flying at $\mathrm{M}=0.80$ ). With a 30-degree crossing angle the range of the two aircraft would be approximately $170 \mathrm{NM} 40$ minutes prior to the loss of separation.

American Institute of Aeronautics and Astronautics 


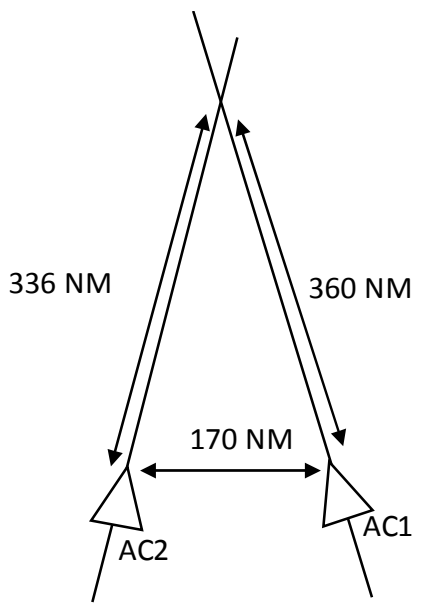

Figure 10. Impact of ADS-B range on intersection track operations

In this example, it is assumed that the current minimum separation available with the ATC automation system is $30 \mathrm{NM}$. The ground automation will report a traffic conflict between these two aircraft. The controller will review the situation to determine the best resolution to the conflict, including whether PTM is a suitable solution. An important part of that decision will be determining when it will be possible to issue the PTM clearance. The ATC automation system will indicate whether PTM is available now or at some later time. With a 30-degree crossing geometry, the earliest time to issue a PTM clearance would be approximately 40 minutes prior to the predicted loss of separation. The controller could then issue a PTM clearance.

If the aircraft are currently further away from the predicted loss of separation (e.g., 60 minutes from the predicted loss of separation), the ground system would indicate to the controller that PTM is not available at that moment but will be available in 20 minutes. The controller would then have the freedom to either wait for 20 minutes before issuing the clearance or decide to use a different approach for resolving the traffic conflict (e.g., issue a flight level change to one of the aircraft if that was available).

\section{Aircraft Sequence Geometries}

PTM operations can involve a number of different geometric orientations. These are based on the number of PTM aircraft and designated aircraft. The most basic PTM geometry involves two aircraft, a PTM aircraft and one designated aircraft. These two aircraft together form a PTM Pair.

When there are more than two PTM aircraft in a sequence and only one designated aircraft at either end of the sequence, these aircraft form a PTM String. Finally, if there are two designated aircraft at either end of a sequence with one or more PTM aircraft between them, that situation is called a PTM Chain. A more detailed description of these geometries is contained below.

1) PTM Pairs - single target geometries

A PTM pair, shown in figure 11, is formed when:

- There are two aircraft in a sequence

- The separation standard between the two aircraft is PTM separation

- There is one PTM aircraft and one designated aircraft
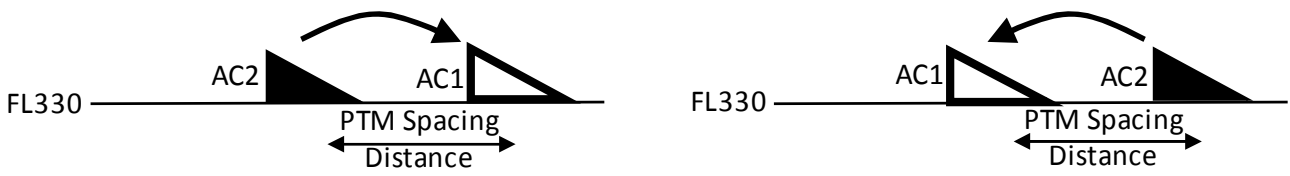

Figure 11. PTM Pairs - single target geometries

American Institute of Aeronautics and Astronautics 
PTM aircraft can be ahead of or behind a designated aircraft. Figure 11 is an example of these different situations. It is important to point out that a designated non-PTM aircraft could be PTM equipped, but it has not been cleared and it is not conducting a PTM operation.

In the figure on the left, the PTM aircraft is behind the non-PTM (designated) aircraft. For the diagram on the right, the PTM aircraft is ahead of the non-PTM (designated) aircraft. The arrow shows the "direction" of the PTM operation.

The obvious effect here is that the behavior of the speed guidance bounds would be reversed. With the PTM aircraft behind the designated traffic, the upper bound would be based on the speed of and distance to the designated aircraft; while if the PTM aircraft were ahead of the designated traffic, the lower bound would be based on the speed of and distance to the designated aircraft.

\section{PTM Strings}

A PTM String is formed when:

- There are more than two aircraft in a sequence

- There is only one designated aircraft in the sequence

- The designated aircraft is leading or trailing the sequence

Figure 12 illustrates two types of PTM strings. The arrows indicate the direction of the PTM operation.

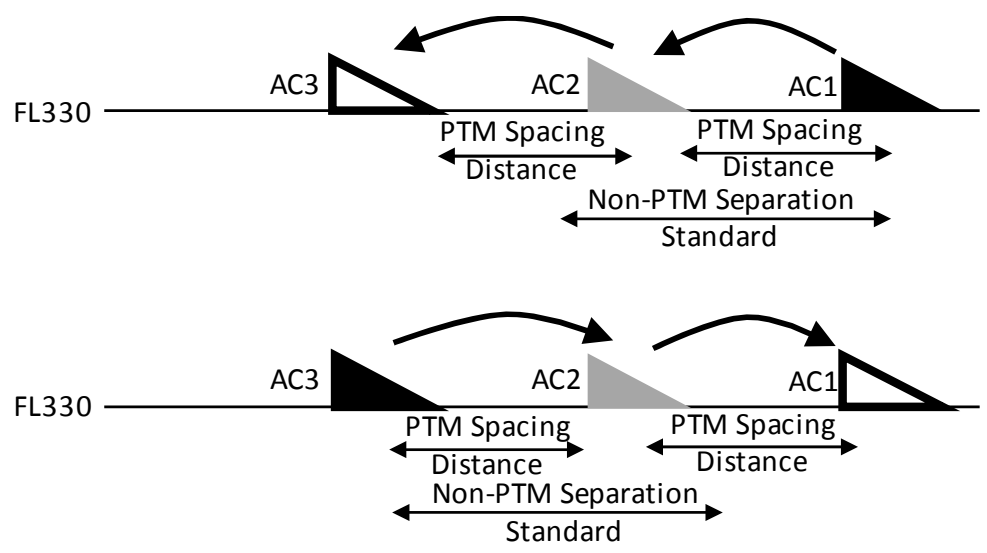

Figure 12. PTM Strings - multiple PTM aircraft, one designated aircraft

\section{PTM Chains}

A PTM chain, shown in figure 13 below, is formed when:

- There are more than two aircraft in a sequence,

- There are two designated aircraft in the sequence, one leading and one trailing,

- The separation standard between the two designated aircraft is a non-PTM separation standard,

- All PTM aircraft in the sequence must be assigned and maintain PTM separation from all designated aircraft in front of and all designated aircraft behind the PTM aircraft (within the non-PTM separation standard distance)

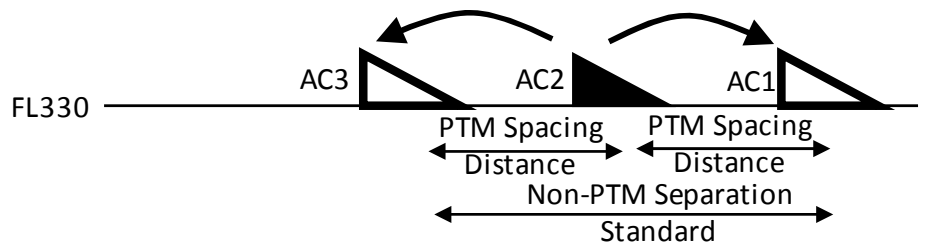

Figure 13. Example of a PTM Chain 
Because of the complexity of the rules necessary for strings and chains, it is anticipated that they will be included in the ground automation system, as well as in the airborne equipment (as needed).

A rule that must be enforced by the ground automation is the independence of PTM operations. That is, the PTM concept does not allow for "interacting" PTM operations. For purposes of interacting operations, a PTM operation is represented by the aircraft contained in a pair, string, or chain. Interacting operations are defined to be when a single designated aircraft is involved in two different PTM operations. For example, an intersecting track PTM operation cannot be performed with an aircraft in a string if at least one of the aircraft would be a designated aircraft for the string and the intersecting track operation.

\section{J. Limit Mach}

As part of a PTM clearance, PTM aircraft are assigned a Limit Mach by ATC. The objective of this Limit Mach is to ensure PTM flight crews are not directed to fly a Mach during a PTM operation that they are unwilling or unable to fly. For trailing operations (where the PTM aircraft is behind the designated aircraft), the Limit Mach would be a minimum Mach number the aircraft could be directed to fly by the FIM equipment. For leading operations (where the designated aircraft is behind the PTM aircraft), the Limit Mach is a maximum Mach number the aircraft could be directed to fly by the FIM equipment. Flight crews will be required to review the assigned Limit Mach and determine if it is acceptable. If the assigned Limit Mach is unacceptable, flight crews should refuse the PTM clearance.

Limit Mach is calculated by the ground automation system based on a set of rules developed for determining Limit Mach for clearances and PTM operations. The following figures help illustrate how Limit Mach is calculated and used during PTM operations.

In figure 14, AC1 is an ADS-B out aircraft flying at FL340 and M=0.80. AC2 is a PTM capable aircraft flying at FL330 and M=0.79. AC2 sends a request to ATC for an altitude change from FL330 to FL340. ATC's ground automation indicates this climb can be supported using PTM. If AC1 does not have a current Mach assignment, ATC sends a Mach assignment clearance to AC1 to maintain 0.80 or greater.

The ground automation then calculates the Limit Mach that would be assigned to AC2. Since this operation is a trailing operation (the designated aircraft, AC1, is ahead of AC2) the Limit Mach is defined to be the minimum of the designated aircraft's assigned Mach or the PTM aircraft's current Mach. For this example, the Limit Mach would be the Mach of the PTM aircraft's current Mach $(\mathrm{M}=0.79)$. ATC sends a PTM clearance to AC2 to follow behind AC1 (while AC2 is still at FL330). The Limit Mach is sent to the aircraft as part of the clearance message.

At the time the clearance is received, the FIM equipment and flight crew evaluate the clearance to determine if the clearance can be accepted. Part of this evaluation includes an evaluation of the ability of AC2 to fly the Limit Mach of 0.79. The flight crew determines if the clearance, including the Limit Mach, is acceptable. The flight crew for AC2 engages the PTM system and the lower bound of the speed guidance is set to $M=0.79$. The upper bound of the speed guidance is determined by the PTM algorithm and is a function of the distance between the PTM aircraft and the current speed of the designated aircraft. The flight crew then accepts the clearance and ATC clears AC2 to climb to FL340. Figure 15 shows the result of receiving the appropriate clearances (which includes the Limit Mach number) and then climbing to FL340.

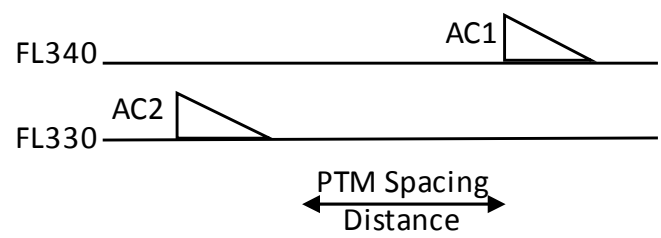

Figure 14. Example of Limit Mach assignment scenario (trailing operation) prior to PTM clearance

American Institute of Aeronautics and Astronautics 


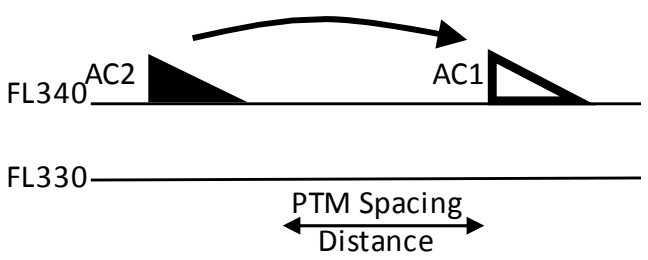

Figure 15. Example of Limit Mach assignment scenario (trailing operation) after PTM clearance acceptance and climb to new altitude

The messages used to support the PTM climb scenario are shown below:

Message Queue [Destination, Sender]

[ATC, AC2] REQUEST CLIMB TO FL340

[AC1, ATC] MAINTAIN MACH 0.80 OR GREATER

[ATC, AC1] WILCO

[AC2, ATC] CLEARED PTM MACH BEHIND AC1, LIMIT MACH FOR PTM OPERATION 0.79

[ATC, AC2] WILCO

[AC2, ATC] CLIMB TO AND MAINTAIN FL340, REPORT REACHING FL340

[ATC, AC2] WILCO

[ATC, AC2] LEVEL FL340

An example of how Limit Mach is determined and used for a PTM operation in a leading operation is shown in figure 16. AC1 is an ADS-B Out aircraft flying at FL340 and M=0.80. AC2 is a PTM capable aircraft, flying at FL330 and $M=0.79$. AC2 sends a request to ATC for an altitude change from FL330 to FL340. ATC's ground automation indicates this climb can be supported using PTM. If AC1 does not have a current Mach assignment, ATC sends a Mach assignment clearance to AC1 (in this case $\mathrm{M}=0.80$ ).

The ground automation then calculates the Limit Mach that would be assigned to AC2. Since this operation is a leading operation (the PTM aircraft is ahead of the designated aircraft) the Limit Mach is defined to be the maximum of the designated aircraft's assigned Mach or the PTM aircraft's current Mach. For this example, the Limit Mach would be the Mach of the designated aircraft's assigned Mach $(\mathrm{M}=0.80)$. ATC sends a PTM clearance to AC2 to conduct PTM ahead of AC1 (while AC2 is still at FL330). The Limit Mach is sent to the aircraft as part of the clearance message.

At the time the clearance is received, the FIM equipment and flight crew evaluate the clearance to determine if the clearance can be accepted, including the Limit Mach of 0.80. The flight crew for AC2 engages the PTM system and the upper bound of the speed guidance is set to $\mathrm{M}=0.80$. The lower bound of the speed guidance is determined by the PTM algorithm and is a function of the distance between the PTM aircraft and the current speed of the designated aircraft. The flight crew then accepts the clearance and ATC clears AC2 to climb to FL340. Figure 17 shows the result of receiving the appropriate clearances (which includes the Limit Mach number) and then climbing to FL340.

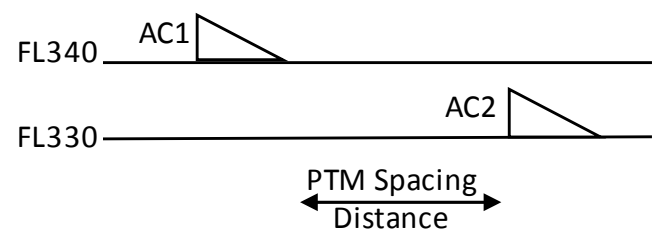

Figure 16. Example of Limit Mach assignment scenario (leading operation) prior to PTM clearance 


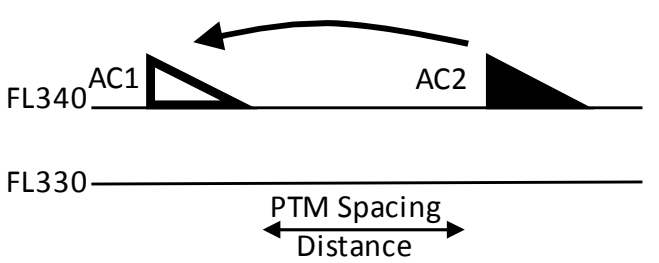

Figure 17. Example of Limit Mach assignment scenario (leading operation) after PTM clearance acceptance and climb to new altitude

\section{Operational Scenarios}

The following scenarios are included to illustrate the concept but are not intended to represent the complete range of possible PTM geometries or supporting systems' functionalities. Each scenario description gives an overview of the operation that is being performed, and what aspects of PTM each scenario is stressing.

\section{A. Same-Track, Co-Altitude Scenario}

In this first scenario, shown in figure 18, the PTM aircraft, operating at FL 330, is overtaking a co-altitude ADSB Out aircraft on the same route.

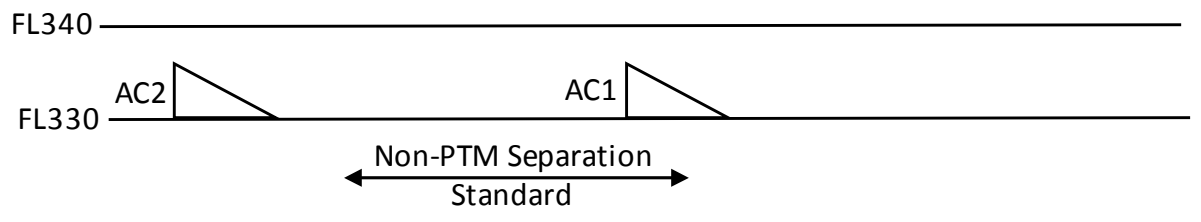

\section{Figure 18. Same-Track, Co-Altitude Scenario}

When the controller determines that a conflict exists and intervention action is required, the controller might issue the overtaking aircraft (AC2) a speed restriction or altitude change to eliminate the overtake and ensure that AC2 gets no closer than a non-PTM separation standard distance.

However, if the ground automation indicates that a PTM clearance is available, the controller would have the option to issue a clearance to AC2 to conduct PTM on AC1 and maintain the current flight level. This clearance would allow AC2 to close to and maintain a distance greater than or equal to the PTM minimum distance with AC1 (figure 19). Since this minimum distance is significantly less than the non-PTM separation distance, AC2 will be able to operate at the desired speed for a longer period of time, increasing the aircraft's overall performance.

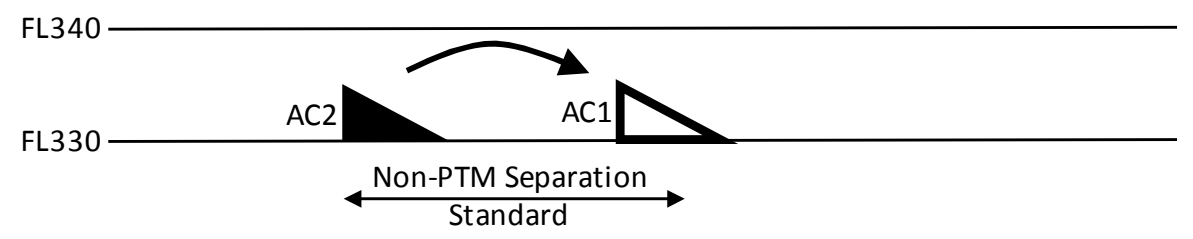

\section{Figure 19. Same-Track, Co-Altitude Scenario}

\section{B. Same-Track, Altitude Change Scenario}

In this scenario, illustrated earlier in the paper, the PTM aircraft is requesting a climb that would cause a conflict with an ADS-B Out aircraft. Initially, it is possible that this will be the most common application of PTM.

General conditions:

- Aircraft are currently vertically separated

- Aircraft are on the same route 


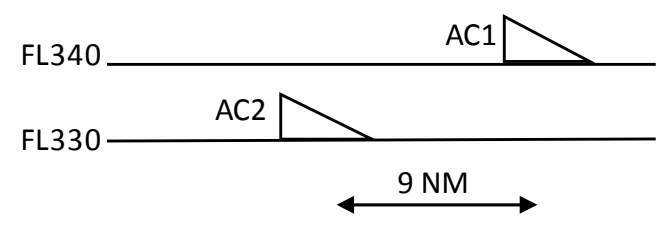

Figure 20. Same-Track, Altitude Change Scenario

Figure 20 shows two aircraft (AC1 and AC2) operating on the same identical track. In this scenario, the crew of AC2 has requested, for operational reasons, an altitude change from FL330 to FL340.

The controller receiving the request would observe that the climb would result in an immediate conflict with AC1 at FL340 using non-PTM separation standard distances. However, because AC2 has indicated that it is PTM equipped (in the flight plan), the ground automation system would indicate the possibility of a PTM clearance (for the controller to issue) that would provide a separation standard that would authorize a climb. The controller would issue a clearance to AC2 instructing it to conduct PTM on AC1.

The clearance information would be loaded into the FIM equipment and the flight crew would confirm acceptability of the PTM clearance. If acceptable, the flight crew engages the FIM equipment, follows the PTM guidance and accepts the clearance with ATC. With the PTM operation engaged, a speed (or speed range) will be commanded by the FIM equipment.

Once the controller has received confirmation that the PTM clearance has been accepted, the controller would then clear AC2 to climb to and maintain FL340. The PTM aircraft would then start a normal climb to FL340. The flight crew follows the speed guidance provided by the FIM equipment throughout PTM operations (figure 21).

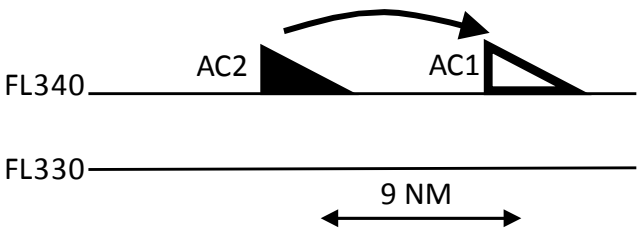

Figure 21. Same-Track, Altitude Change Scenario

\section{Same-Track Loading, Multiple Aircraft Interactions (Track Loading)}

It is anticipated that a significant consideration in the benefit case will be the ability to have multiple, co-altitude, same identical track aircraft operating with different equipage levels and a PTM separation standard applied between aircraft. This is particularly beneficial when "loading" aircraft on fixed routes and organized track systems. This track loading occurs as aircraft transition from radar surveillance airspace to oceanic, non-radar airspace. The required spacing based on non-PTM separation distances often limits the number of aircraft that can enter a given track at a given altitude. There are times when aircraft must fly at less than optimal altitudes or fly different tracks. The reduced spacing due to PTM will mean that more aircraft will be able to fly where and when they want to for better aircraft and system performance.

This is illustrated in figure 22 below. In this case, there are three aircraft that would like to operate on the same oceanic track at similar times. As seen in the left graphic of figure 22, without PTM, controllers would have to put the second aircraft on a different track or at a different altitude in order to prevent a traffic conflict. As seen in the right hand graphic of figure 22, using PTM, the second and third aircraft can be instructed to use PTM and all three aircraft can operate on their desired track at their desired altitude. 

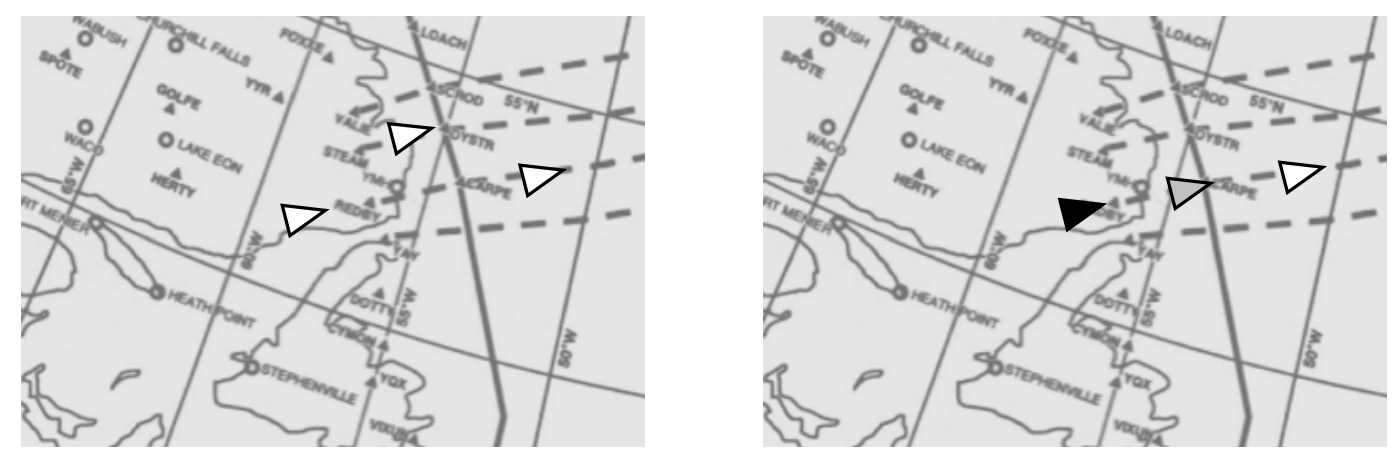

Figure 22. Example of how PTM could be used to improve track loading

\section{Intersecting Tracks}

PTM is also applicable for intersecting track situations. In general, there will be restrictions on the maximum possible crossing angle permitted by PTM. As stated earlier, this is often due to limitations of the ADS-B signal range, and the requirement that the controller resolve conflicts by some time or distance prior to the actual loss of separation. Typically, this will restrict the intersecting track angle to about 30 degrees.

\section{Crossing Path Scenario}

For the crossing shown in figure 23, aircraft AC2 and AC1 are moving towards a loss of separation in an oceanic environment (30 NM minimum separation). The aircraft are both at FL310 and Mach 0.80. The ground automation system is reporting that a loss of separation will occur in 40 minutes, when the distance between the two aircraft will decrease to less than $30 \mathrm{NM}$. The controller may choose to use a PTM clearance to resolve the conflict.

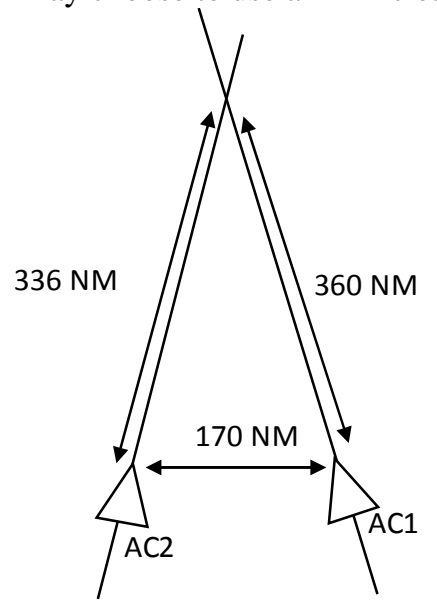

Figure 23. Example of PTM operations with intersecting tracks

When the PTM clearance is received by AC2, the PTM clearance information would be loaded into the FIM equipment. The acceptability of the PTM clearance would be checked against associated criteria required for PTM operations. The FIM equipment uses data received from AC1 and the ground automation system to predict the time, location, and separation distance at the closest point of approach. This distance is called the projected closest distance (PCD). This displayed distance is a continuously updated value of the closest distance that the PTM aircraft will get to the position of the designated aircraft. It is computed based on the current commanded speed of the PTM aircraft and the current speed of the designated aircraft. As such, when the flight crew changes the aircraft's command speed, the PCD will change immediately. If the traffic situation is such that the distance at the closest point of approach is less than the PTM-enabled spacing minimum, the FIM equipment displays, to the flight crew, speed guidance (different from the current speed) that would ensure the required spacing between the PTM aircraft and the designated aircraft is satisfied during the PTM operation. If the proposed guidance was acceptable, the flight crew would engage the FIM equipment and accept the clearance. It is likely that, while the aircraft will pass too close for a non-PTM separation standard, the aircraft will be significantly farther apart than required for the PTM separation standard. The flight crew follows the speed guidance provided by the FIM equipment throughout PTM operations (figure 24).

American Institute of Aeronautics and Astronautics 


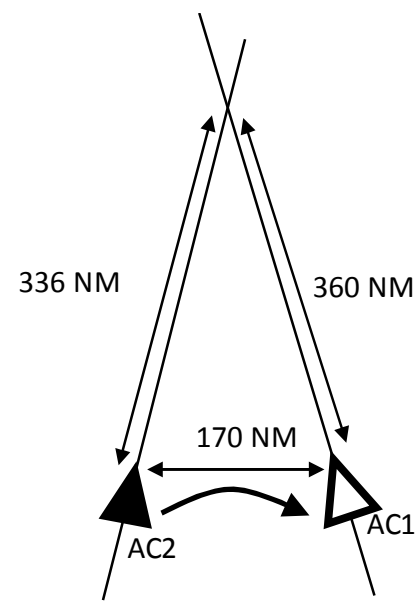

Figure 24. Example of PTM operations with intersecting tracks

Once the aircraft have passed the closest point of approach and the controller determines that a non-PTM separation standard has been achieved (e.g., $30 \mathrm{NM}$ ), the controller may terminate the PTM procedure.

\section{Merging Path Scenario}

PTM can also be utilized when the paths of two aircraft merge. This could happen with an aircraft joining an organized route structure at a waypoint along the route as illustrated in figure 25 below.

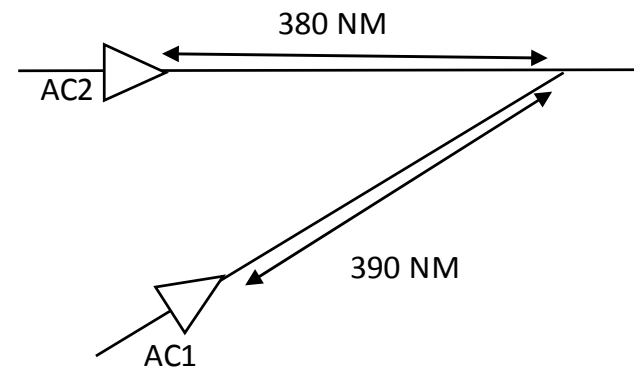

\section{Figure 25. PTM merging track scenario}

In this scenario, the controller predicts there would be a loss of separation if AC1 joined the track of AC2 using non-PTM separation standards. Without PTM, the controller would normally have to change the altitude or significantly change the speed of either AC2 or AC1. However, using PTM, the controller can avoid the altitude or significant speed change, and issue a PTM clearance to the PTM aircraft (figure 26). The clearance message would include the expected relative position the controller is expecting for the operation (e.g., "ahead of" or "behind"). In the case above, the clearance to AC2 would be similar to "CLEARED PTM MACH, MERGE AHEAD OF AC1".

380 NM

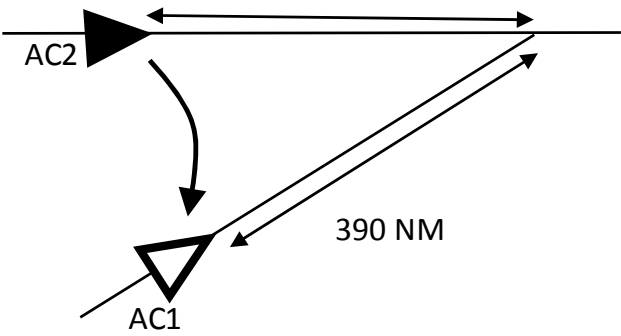

Figure 26. PTM merging track scenario

American Institute of Aeronautics and Astronautics 
While engaged, the FIM equipment compares the trajectory of the PTM aircraft with the location and planned trajectory of the designated aircraft, and provides speed commands that are predicted to result in avoiding a loss of spacing.

In the above example, the pair of aircraft will transition to an in-trail maneuver invoking use of the functionality described in earlier scenarios. Once established in the in-trail operation, the FIM equipment will continue to perform a PTM operation until a pair-specific terminate message is received from ATC. This could happen after route divergence, if vertical separation is established, or if a speed difference increases the distance between the PTM aircraft and the designated aircraft to a distance where a non-PTM separation standard can be applied.

\section{Ground Automation and Air Traffic Controller Procedures}

\section{A. Ground Automation}

Currently, US-controlled oceanic airspace is managed with the Advanced Technology and Oceanic Procedures (ATOP) system (which includes the Ocean 21 system and controller interface) ${ }^{2}$. This system gathers aircraft position information either through ADS-C position reports or High Frequency (HF) voice position reports. Using this information and the aircraft's flight plan, the system projects the future position of each aircraft. The system includes a conflict probe that is used to identify potential traffic conflicts up to two hours in the future. The separation standard that is applied for conflict detection may vary by aircraft, and is a function of the type, frequency, and/or quality of the position reports received from each aircraft as well as aircraft equipage.

Losses of separation (conflicts) that are projected to occur within two hours but greater than 30 minutes into the future are displayed to the controller with an advisory conflict alert indication. Conflicts displayed to the controller that will not be resolved within 30 minutes prior to loss of separation are given a higher level of alerting on their display. The ATOP system displays a list of all active (unresolved) conflicts.

The Ocean 21 system uses intent information on all aircraft (via the flight plan) to predict future trajectories. It also provides a method of communication between the controller and flight crew. Finally, as appropriate clearances are input into the system, it removes resolved conflicts from the conflict list. All three of these capabilities will be utilized in supporting PTM operations.

Many other automation systems used throughout the world to manage oceanic airspace have similar characteristics. PTM operations will require significant integration with the controller's ground automation system. Some of the anticipated ground system functionality should include:

- Identify potential PTM situations.

- Formulate clearances for the controller to issue.

- Send intended flight path information of designated aircraft to PTM aircraft.

- Recognize accepted PTM clearances (and consider designated conflicts resolved).

- Identify when non-PTM separation is re-established.

- Notify the controller that PTM is no longer required, and be able to send a pair-specific PTM cancellation message to the FIM equipment.

\section{B. Air Traffic Controller}

Within the current oceanic system, the controller is tasked with maintaining separation between all participating aircraft in their assigned airspace. This is accomplished by having a method of determining aircraft position and projected positions (position reports and ground automation support), and applying rules, techniques, and skill to identify conflicts in a timely manner and resolve them prior to a loss of separation.

PTM operations begin once a controller has identified that a traffic conflict will exist and when a controller determines that the PTM separation standard is applicable and beneficial for the safe and efficient operation of the airspace. The ground automation system identifies the traffic conflict and the controller determines which separation standard is most applicable.

If PTM is the most applicable or preferred option, the controller will issue a PTM clearance. Once accepted, the flight crew is then required to manage speeds based on information provided by the FIM equipment.

The controller also has the option of assigning additional designated aircraft to aircraft already conducting PTM operations. The controller accomplishes this by sending a new clearance to the PTM aircraft. This new clearance instructs the flight crew to continue doing PTM on the aircraft already assigned to it and includes instructions to conduct PTM on the additional designated aircraft.

American Institute of Aeronautics and Astronautics 
Similarly, if the controller no longer prefers to use PTM between the PTM aircraft and a subset of the already designated aircraft, the controller will send a new clearance to the PTM aircraft. This clearance will identify which PTM operations should be continued and which are no longer required or desired.

To make use of the PTM clearance, the controller is responsible for:

- Determining if a PTM clearance is suitable, including PTM equipage.

- Determining and verifying the PTM aircraft and the designated aircraft call signs.

- Ensuring separation between the PTM aircraft and all other aircraft, excluding the designated aircraft.

- Ensuring that the designated aircraft is not authorized to deviate from its cleared flight plan during the duration of the PTM maneuver.

- Terminating the PTM operation after non-PTM separation is re-established and when the controller no longer desires to apply the PTM clearance.

\section{Controller Monitoring of Procedure Compliance}

During the development of the PTM concept, a one-way data link between the ground automation and the FIM equipment was assumed. In other words, the ground automation has the ability to communicate with the FIM equipment, but the FIM equipment has no means of sending data link messages to ground automation. If it were possible and practical to remove that constraining assumption, so that the avionics could have a two-way data link with the ground system, then there are some possible benefits that could be realized.

Due to communications and surveillance limitations, ATC currently uses procedural separation in oceanic airspace. However, in airspace in which aircraft spacing is guaranteed with procedural separation, it is not uncommon for controllers to monitor the aircraft compliance with the procedure (not the spacing between aircraft but compliance with the procedure). A very common example of this is ground system alerting to deviations from an assigned altitude and route. Whether or not there is an associated loss of aircraft separation, the controller monitors and reacts to deviations from the assigned procedural clearance. This same concept could be valuable in PTM procedural operations.

When a PTM clearance is issued and accepted, the ground system could receive reports on the FIM equipment health, the status (engaged in a PTM operation vs. not engaged in a PTM operation), target status (loss of signal), and certain flight crew alerts that the flight crew has not responded to within an appropriate interval. This monitoring would improve the PTM overall safety performance by reducing the impact of any flight crew training and performance issues, and provide ground monitoring of avionics system health.

Actual and projected aircraft spacing information would not be data linked to the controller. The controller is neither trained nor capable of applying tactical separation with aircraft involved in PTM.

This down link data channel could include the following standard message from the FIM equipment:

- $\quad$ PTM state [Pending PTM operation, Pending PTM operation/Engaged PTM operation, Engaged PTM operation, no activity]

- $\quad$ Target Status

- Call sign of all PTM aircraft engaged in a PTM operation

- Any (and all) alerts of a moderate to high severity level that are displayed to flight crew

A new message would be sent if there is a change in any of the fields

\section{Flight Deck Automation and Flight Crew Procedures}

\section{A. Flight Deck Automation}

The FIM equipment will need numerous functions certified. Some of the anticipated functions include:

- The ability to receive PTM clearance data from the ground system.

- Status monitoring and appropriate notification of status of the FIM equipment.

- Interface with the flight crew to enable the flight crew to follow Mach guidance to support PTM operations.

- Adequate alerting to the flight crew of failure to comply with Mach guidance

- Alerting to the flight crew of an imminent loss of spacing or loss of spacing.

- Alerting to the flight crew of a loss of separation

\section{B. Flight Crew Procedures}

In non-PTM operations, the flight crew's responsibility (role) in maintaining traffic separation is to comply with the assigned route, altitude, and speed (as necessary). The change with PTM is that the Mach guidance is generated

American Institute of Aeronautics and Astronautics 
by on-board FIM equipment rather than provided by the controller. The significant job responsibility change (or addition) is the requirement for the flight crew to be familiar with and able to execute responses to PTM alerts and equipment failures.

When a controller issues a PTM clearance to a flight crew, the flight crew of the PTM aircraft will use their ADSB-enabled on-board FIM equipment to manage their speed to achieve spacing relative to the aircraft designated by the controller. The flight crew task of flying the assigned altitude, route, and speed does not change, with the exception that the required speed (Mach) is determined by the FIM equipment and authorized by the PTM Mach clearance. Regarding speed, the flight crew is responsible for the task of following speed guidance from a new source (FIM equipment vs. controller).

When the flight crew receives any PTM clearance, the flight crew is responsible for reviewing the proposed PTM Mach guidance (fixed Mach speed or Mach speed range) to determine if the proposed guidance would be operationally acceptable. The flight crew would also review other information that could influence their decision to accept or reject the PTM clearance. This would include assessing potential future speed restrictions (e.g., the eventual speed the PTM aircraft may be required to fly due to the speed of the other aircraft) based on the current speeds of the designated aircraft. Other information that could influence the flight crew's decision would include aircraft performance, weather conditions, available ride information, and general traffic situation awareness.

The guidance (along with suitable alerting) will be provided to the flight crew with an interface function that resides either within the current displays, or through an auxiliary display function.

The PTM operation puts additional responsibilities on the flight crew of the PTM aircraft. They include:

- Validating the operational status of the FIM equipment.

- Rejecting the PTM clearance if, in their sole discretion, the outcome will be operationally undesirable.

- Being familiar with and prepared to execute appropriate maneuvers should the FIM equipment fail (failure could include the designated aircraft's ADS-B transmitter failing).

- Being familiar with and able to execute responses to PTM alerts.

- Complying with guidance displayed by the FIM equipment.

- Acknowledging instructions from the controller that terminate the PTM operation and resuming normal operations.

\section{Airborne Human-Machine Interface for PTM}

\section{A. Airborne PTM Human-Machine Interface}

Flight crews authorized to conduct PTM operations will require the use of a flight crew HMI. Future implementations of an airborne HMI that will support PTM operations may be fully integrated, for example, with an aircraft's primary flight display, navigation display, and multifunction control display unit. However, another possible implementation involves the flight crew HMI operating independently of other flight guidance and navigation displays. A non-integrated implementation of this type, which may be considered for retrofit installations, was designed and investigated at the NASA Langley Research Center. ${ }^{3,4}$

NASA Langley's airborne PTM HMI consists of two primary hardware components - a side mounted display with a touchscreen interface and a $7.62 \mathrm{~cm}$ x $7.62 \mathrm{~cm}$ Configurable Graphics Display (CGD) located in the flight crew's primary field-of-view (FOV). Possible locations for left-seat PTM HMI components within the flight deck are shown in figure 27.

The flight crew will use the side mounted display to review PTM clearances, engage or clear the FIM equipment, review displayed messages, indications, and alerts, and as a situation awareness display tool. The CGD will display the PTM guidance as well as indications that advise the flight crew to review the side mounted display. The CGD is located in the flight crew's primary FOV and is intended to be the primary display used to ensure conformance with PTM guidance during PTM operations. As mentioned previously, CPDLC is also assumed to be in use during PTM operations.

American Institute of Aeronautics and Astronautics 


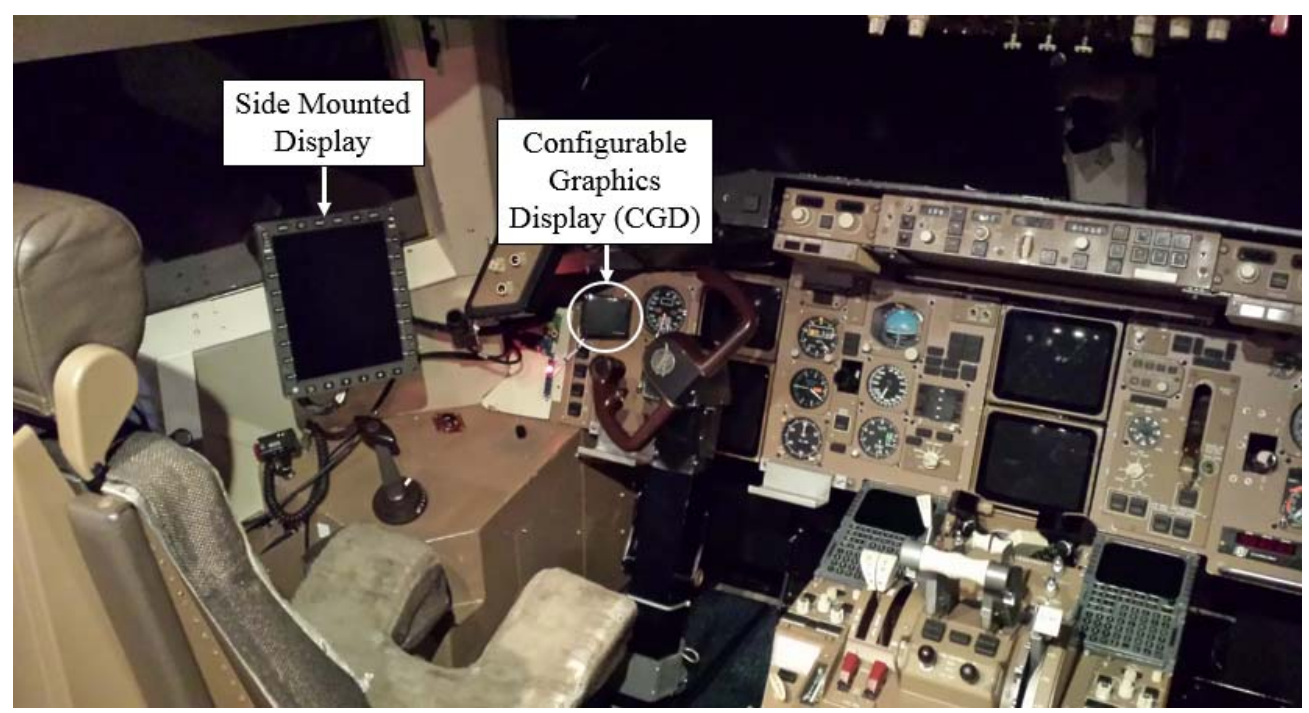

Figure 27. Possible locations for left-seat PTM HMI components within the flight deck

\section{B. Side Mounted Display}

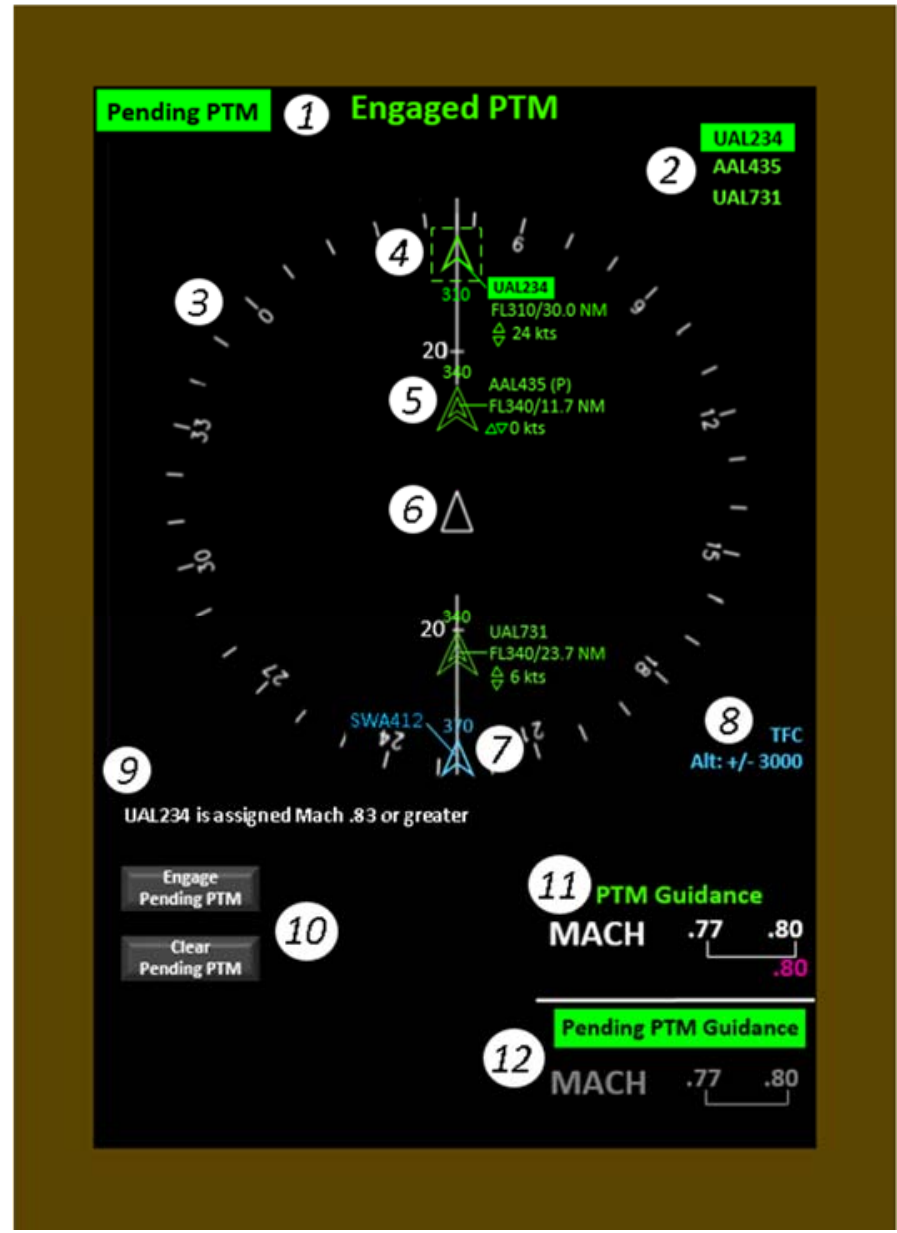

1 - PTM Mode Indicator. Displays current status of PTM. Can display one of the following five modes:

- "Pending PTM"

- "Engaged PTM"

- "Pending PTM" \& "Engaged PTM"

- "Cancelled PTM"

- "NO PTM"

2 - Flight Identification (ID) List. Displays flight ID of all aircraft that are or may become engaged in a PTM operation with the PTM aircraft. Normal text indicates designated aircraft. Reverse video text indicates pending aircraft. Aircraft flight ID's are listed in the order that aircraft symbols appear on the cockpit display of traffic information (CDTI), from top to bottom.

3 - Cockpit Display of Traffic Information (CDTI). Displays ownship symbol and ADS-B traffic that is within ADS-B signal range. Current heading is shown in the compass rose located around the perimeter of the CDTI. Distance is shown by the tick marks on the center lubber line. Various range scales may be selected.

Figure 28. Side mounted display. 
4 - Pending Aircraft Symbol and Data Tag. Each pending aircraft is displayed as a single green chevron surrounded by a dashed box. Flight ID is shown in reverse video text. The rest of the data tag is shown in green text.

5 - Designated Aircraft Symbol and Data Tag. Each designated aircraft is displayed as a double green chevron. The information in data tags associated with designated aircraft can be reduced by the pilot, leaving only the flight ID.

6 - Ownship Aircraft Symbol. Ownship is displayed as a hollow white triangle.

7 - Background Aircraft Symbol and Data Tag. Each ADS-B Out aircraft not being used by ownship as pending or designated aircraft in a PTM operation is displayed as a single cyan chevron. Only the flight ID is included in a background aircraft's data tag, and the flight ID can be removed by the pilot.

8 - Background Traffic Indicator. Shows the altitude filter applied to determine which background aircraft are displayed. The setting for the Altitude Filter is changed within the Traffic Display menu. The Background Traffic Indicator serves as a selectable area of the display screen and may be used to toggle background traffic off/on. When background traffic is off, TFC OFF is displayed.

9 - Message Area. PTM related messages are displayed in this area. White text is associated with information and indications; amber text is associated with cautions; and white text with red highlighting is associated with warnings.

10 - Selectable Buttons. Various buttons, used to interact with the FIM equipment, are displayed depending on the PTM mode. Two columns of buttons are displayed during the "Engaged PTM" mode (in contrast to the combined "Pending PTM / Engaged PTM" mode shown above). During an engaged PTM operation, additional data associated with a flight crew selected aircraft may be displayed in a data block that replaces the right column of buttons. The right column of buttons can also be replaced with a checklist during rare non-normal events.

11 - PTM Guidance. Displays Mach guidance (in white) that ownship is allowed to fly during Engaged PTM mode. Flight crew selected Mach for ownship is shown in magenta. During Engaged PTM mode, this area "repeats" the PTM guidance shown on the CGD. When areas 11 and 12 display the exact same information as that shown on the CGD, areas 11 and 12 of the side mounted display are segregated by white border lines on the top and left.

12 - Multi-Use Information Area. Displays information pertaining to PTM, but is normally blank. During the Pending PTM mode, "Pending PTM guidance" is displayed (in grey) to show the flight crew the PTM guidance that the PTM aircraft will be required to fly if a pending PTM clearance is accepted. There are times, when the FIM equipment determines that PTM conditions are not met when ATC issues a PTM clearance. When this occurs, the words "Unable Pending PTM" are shown (in white) since the option to accept the PTM clearance is not available to the flight crew. Other than in a Pending PTM mode, the word "Message" is displayed in area 12 when a message is shown in the Message Area. Additionally, when areas 11 and 12 display the exact same information as that shown on the CGD, areas 11 and 12 of the side mounted display are segregated by white border lines on the top and left.

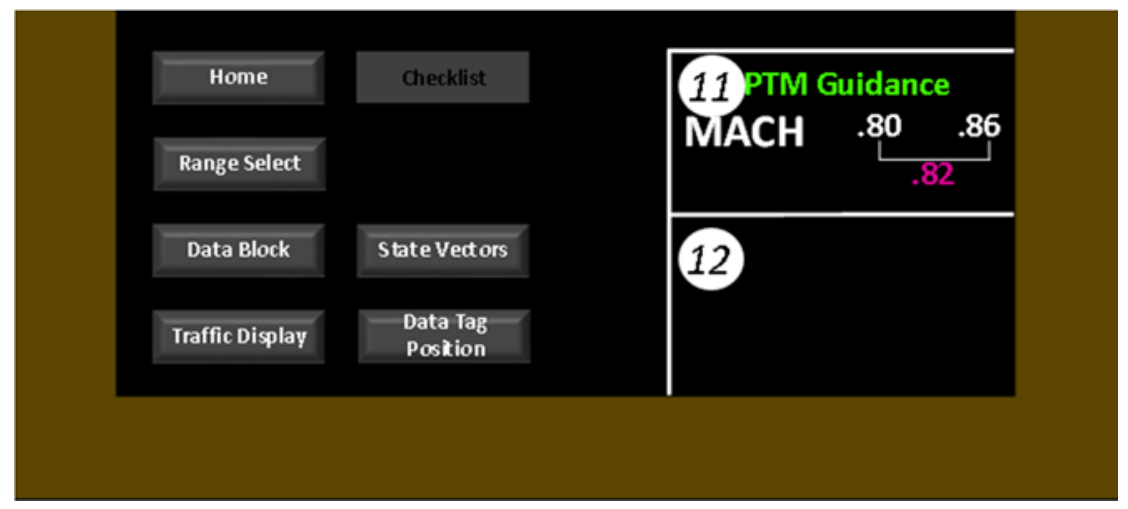

Figure 29. Multi-use information area.

American Institute of Aeronautics and Astronautics 


\section{Configurable Graphics Display (CGD)}

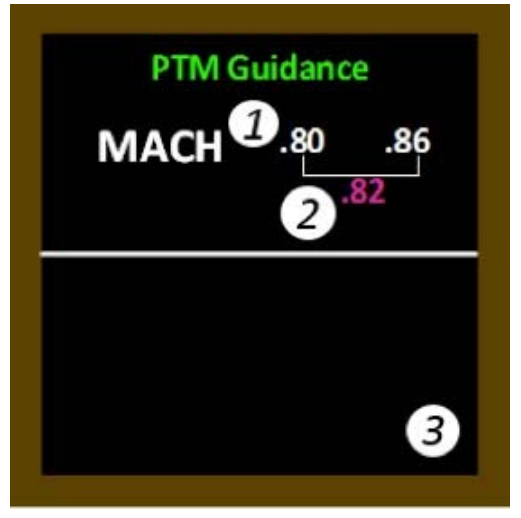

Figure 30. Configurable Graphics Display.
1 - PTM Guidance (shown in white) that ownship must fly during a PTM operation.

2 - Flight Crew Selected Mach (shown in magenta). This may be from the mode control panel (MCP) or an available mode of the flight management computer (FMC). When the flight crew selected Mach is set at a PTM guidance range limit, at one end or the other, the flight crew selected Mach will be displayed under that limit. Otherwise, the flight crew selected Mach will be displayed under the center of the range.

3 - Multi-Use Information Area. Displays information pertaining to PTM operations, but is normally blank. "Pending PTM" will be displayed in green reverse video during a Pending PTM mode. "Message" will appear whenever a message is present on the side mounted display in the message area. The color of the "Message" notification will match the color of the message displayed.

The CGD presents PTM guidance within the flight crew's primary FOV and presents notifications that direct flight crew attention to information located on the side mounted display.

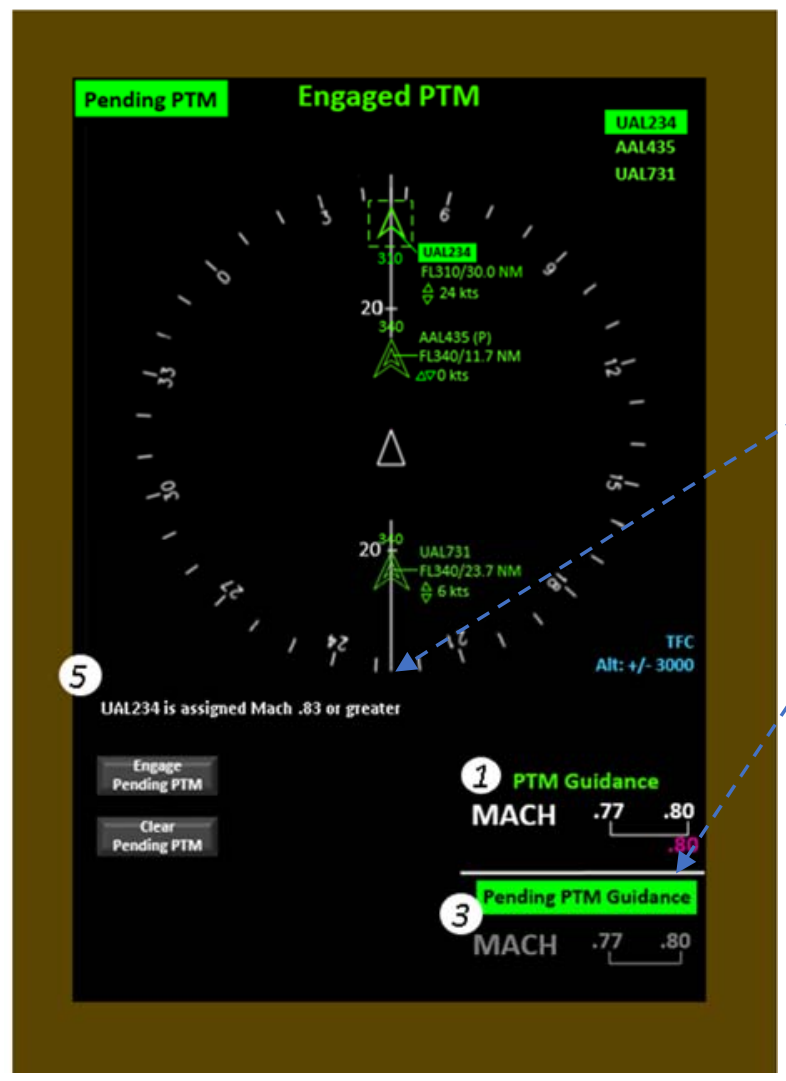

Figure 31. Relationship between the side mounted display and the CGD

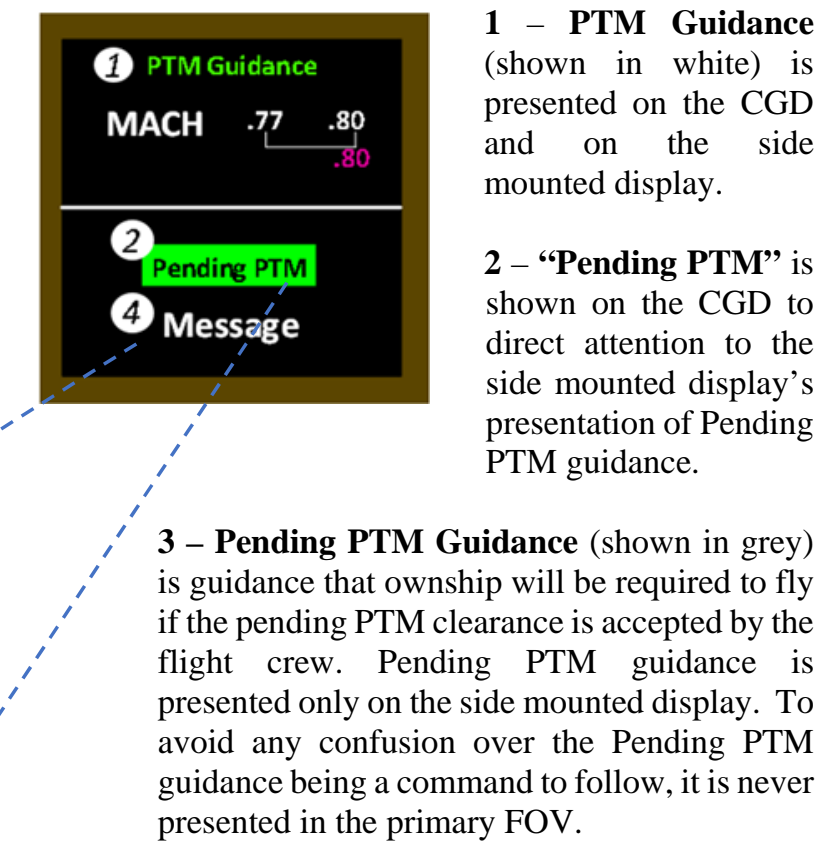

4 - "Message" will appear on the bottom of the CGD any time there is information presented in the side mounted display's Message Area.

5 - Message Area has a larger space available to provide information and indications to the flight crew.

References 3 and 4 provide much more detail on the design, development and evaluation of the NASA Langley developed flight crew HMI. 


\section{Benefits Analysis}

\section{A. Experiment Design}

A fast-time experiment to support the development and evaluation of the PTM concept of operations was conducted at the NASA Langley Research Center. The focus of this experiment was to evaluate the benefits of PTM in the Central East Pacific (CEP) oceanic airspace region. This experiment considered the parameters of ADS-B In equipage, FIM equipment, PTM separation standard and airspace separation standard. ADS-B In equipage was varied as a percentage of the aircraft in the airspace, with the settings of $10 \%, 20 \%, 45 \%, 70 \%$, and $80 \%$ being used. It was assumed that all aircraft in the airspace would be equipped with ADS-B Out, given the Federal Aviation Administration (FAA) mandate on ADS-B Out equipment being installed by 2020. The PTM separation standard, which was set to 5 or $10 \mathrm{NM}$ in the experiment, was only applied to aircraft that were equipped with PTM. The final parameter of airspace separation standard, which was applied to non-PTM equipped aircraft, was 15, 30, or 50 NM. To model the density and distribution of aircraft within the CEP, recorded traffic data were received from the FAA and converted into scenario files that could be used in the simulation. This allowed for the experiment to use traffic patterns that are comparable to what aircraft in real operations would experience. A nominal unconstrained airspace was also examined, in which every aircraft was able to fly the exact altitudes that were desired at all times. This provided an upper bound to the maximum benefits possible in the CEP airspace region.

\section{B. Sample Results}

The data from this experiment were analyzed to observe the benefits experienced by unequipped aircraft and by PTM equipped aircraft. Several measures were compared between the current day airspace, PTM test case airspace, and the unconstrained airspace to quantify the benefit of PTM. Aircraft equipped with PTM resolved more conflicts which led to improvements in fuel efficiency and increased time on optimum flight level.

A main interest during this study was the difference in fuel efficiency, expressed in nautical miles per thousand pounds of fuel as well as percent difference. It is a measure of change in fuel performance between corresponding aircraft in different airspaces. Table 2 below shows the average percent differences in fuel efficiency above current day for PTM equipped aircraft, unequipped aircraft in the PTM airspace, and the unconstrained airspace. Unequipped aircraft average essentially no difference over current day while unconstrained and PTM aircraft see improvements. In fact, PTM equipped aircraft see improvements that nearly reach the values gained by unconstrained aircraft. PTM equipped aircraft are predicted to experience a $0.93 \%$ increase in fuel efficiency over current day airspace operations (assuming a 50NM non-PTM separation standard distance).

\begin{tabular}{|l|c|c|c|}
\hline \multirow{2}{*}{ Airspace } & \multicolumn{3}{|c|}{ Current Day, non-PTM separation } \\
\cline { 2 - 4 } & $\mathbf{1 5} \mathbf{~ N M}$ & $\mathbf{3 0} \mathbf{~ N M}$ & $\mathbf{5 0}$ NM \\
\cline { 2 - 4 } & Mean (\%) & Mean (\%) & Mean (\%) \\
\hline $\begin{array}{l}\text { PTM Airspace: } \\
\text { Unequipped Aircraft }\end{array}$ & 0.02 & 0.04 & 0.06 \\
\hline $\begin{array}{l}\text { PTM Airspace: } \\
\text { Equipped Aircraft }\end{array}$ & 0.84 & 0.88 & 0.93 \\
\hline Unconstrained & 0.88 & 0.92 & 0.97 \\
\hline
\end{tabular}

Table 2. Mean percent difference in fuel efficiency over current day by airspace.

For more detailed information on this experiment and the results obtained, see reference 5 .

\section{Summary}

Pairwise Trajectory Management is an Interval Management concept that utilizes airborne and ground-based capabilities to enable and benefit from airborne pairwise spacing capabilities in oceanic regions. The goal of PTM is to use airborne surveillance and tools to manage an "at or greater than" inter-aircraft spacing. Due to the precision of ADS-B information and the use of airborne spacing guidance (speed commands), the PTM minimum spacing distance will be less than distances a controller can support with current automation systems that support oceanic operations.

American Institute of Aeronautics and Astronautics 
Ground tools assist the controller in evaluating the traffic picture and determining appropriate PTM clearances to be issued. Avionics systems provide guidance information that allows the flight crew to conform to the PTM clearance issued by the controller. The combination of a reduced minimum distance and airborne spacing management will increase the capacity and efficiency of aircraft operations at a given altitude or volume of airspace.

This paper provides an overview of the proposed application, description of a few key scenarios, and high level discussion of expected air and ground equipment and procedure changes, a description of a proposed flight crew human-machine interface and some initial benefits analyses. A more detailed description of the PTM concept of operations can be found in reference 6.

\section{References}

${ }^{1}$ Jones, K. M., “Pair-Wise Trajectory Management-Oceanic (PTM-O): Concept of Operations-Version 3.9,” NASA TP-2014218188, 2014.

2 “Operator's Manual, Air Traffic Controller’s Manual: Advanced Technologies and Oceanic Procedures, Model - T24.00”, NASP-4508G, U. S. Department of Transportation, Federal Aviation Administration, 2016.

${ }^{3}$ Kibler, J. L., Chartrand, R. C., Graff, T. J., and Jones, K. M., "Design of an Airborne Human-Machine Interface for Interval Management (IM) Pairwise Trajectory Management (PTM),” NASA TM in preparation.

${ }^{4}$ Kibler, J. L., Chartrand, R. C., Graff, T. J., and Jones, K. M., "Pairwise Trajectory Management (PTM) Pilot Training Requirements Focus Group and Human-in-the-Loop (HITL) Testing Activity,” NASA TM in preparation.

${ }^{5}$ Chartrand, R., and Ballard, K., "Benefits of using Pairwise Trajectory Management in the Central East Pacific", 2017 AIAA Aviation and Aeronautics Forum and Exposition, Denver, CO, 2017.

${ }^{6}$ Jones, K. M., Graff, T. J, Chartrand, R. C., Carreño, V. C., and Kibler, J. L., "Pairwise Trajectory Management (PTM): Concept Overview”, NASA TM in preparation. 